\title{
The failure of the monetary model of exchange rate determination
}

\author{
Dinçer Afat ${ }^{\mathrm{a}}$, Marta Gómez-Puig ${ }^{\mathrm{a}}$ and Simón Sosvilla-Rivero ${ }^{\mathrm{b}^{*}}$ \\ ${ }^{a}$ Department of Economic Theory, Universitat de Barcelona. 08034 Barcelona, Spain \\ ${ }^{b}$ Complutense Institute for International Studies, Universidad Complutense de Madrid. 28223 Madrid, \\ Spain
}

March 2015

\begin{abstract}
In this paper, we test three popular versions of the monetary model (flexible price, forward-looking and real interest differential models) for the OECD member countries by applying Johansen cointegration technique. Based on country-by-country analysis, we conclude that monetary models do not provide the expected results. We reveal several shortcomings of the models and examine the building blocks of the fundamental version. Although researchers always blame the deviations from purchasing power parity as the reason for the failure of the monetary model, our analysis indicates that invalidity of Keynesian money demand function is also responsible for unfavourable results.
\end{abstract}

JEL Codes: F31, F41

Keywords: exchange rate, flexible price monetary model, forward-looking monetary model, real interest differential model, money demand, purchasing power parity

"Corresponding author. Tel.: +34 913942342; fax: +34 913942591.

E-mail addresses: dafatafa7@alumnes.ub.edu (D.Afat), marta.gomezpuig@ub.edu (M.Gómez-Puig), sosvilla@ccee.ucm.es (S.Sosvilla-Rivero) 


\section{Introduction}

Exchange rate modelling is very crucial not just for economic theory but also for financial practitioners. The search for an acceptable model to explain the movements of exchange rates with macroeconomic variables has led to an extensive literature on exchange rate modelling (see, e. g. Sarno and Taylor, 2002 or MacDonald, 2007 for a survey). This theoretical literature does not rest on a fully specified macroeconomic framework that captures all the main exchange rate influences and transmission mechanism, attention being focused on certain relationships while excluding others.

The popular framework; balance of payments approach, focuses on the flows of currencies arising from international transactions for goods, services, portfolio investment and direct investment; whereas the asset market approach emphasises outstanding stocks of currencies as well as the current determinants of money demands. The asset market approach treats money as an asset; accordingly, exchange rates are asset prices. Within the asset market approach, the monetary model, which applies supply and demand mechanism to determine the exchange rates, attracts much attention both from the theoretical and empirical points of view.

A vast literature on exchange rate modelling has developed to offer empirical evidence on the relevance of the monetary model variants. The general conclusion from this literature is mixed and inconclusive: the models seemed to work, to some extent, for the first period of the floating experience (i. e., 19751978), but they have not worked so well for the following decades. The results also depend on the estimation techniques. Recent studies based on panel data estimations provide more support for the models (see, e.g. Cerra and Saxena, 2010 for panel cointegration results), bringing up the discussion on the validity of the monetary model again. Consequently, it is considered whether the negative results are due to insufficient econometric techniques. Therefore, researchers employ various new methods for estimations and compare the findings.

Meese and Rogoff (1983a, b) and Cheung et al. (2005) focus on the explanatory power of the monetary models and state that it is extremely poor, not performing better than a naive random-walk model in the post sample forecasting tests, even though realised explanatory variables are used during the post sample period. Whereas MacDonald and Taylor (1994) assert that an unrestricted version of monetary model outperforms the random walk and some other models in an out-of-sample forecasting contest. Yet, we can say that unfavourable results outweigh the favourable ones. As Neely and Sarno (2002) put it: "...the negative results for monetary models have nonetheless produced a conventional wisdom in the profession that exchange rate changes cannot be forecast-or cannot be forecast using macroeconomic fundamentals. It is not clear, however, that this is true."

Although monetary model variants have been tested many times, reasons for the failure of the models have not been extensively investigated. Thus, the main objective of this paper is to offer further empirical evidence and explanations on the failure of the monetary model. Our work contributes to the literature in several ways. We reveal various shortcomings of the model that are not mentioned in the previous analyses. We employ a wide range of currency pairs (consist of OECD member countries' 
currencies), which comprise major reserve currencies that are commonly used in the empirical literature as well as the remaining (non-reserve) currencies that are generally neglected, probably due to the limited trade turnover and low level of international demand. Our research covers three popular variants of the monetary model together with the building blocks of the fundamental version.

The rest of this paper is structured as follows. Section 2 outlines the theoretical framework and provides our criticisms. Section 3 describes the data and the econometric methodology adopted in this study. Section 4 reports the empirical results, and Section 5 offers some concluding remarks.

\section{Theoretical Framework}

\subsection{Flexible Price Monetary Model}

The Flexible Price Monetary Model (FPMM) or the Frenkel-Mussa-Bilson ${ }^{1}$ model rests on two basic assumptions. First, all prices are assumed to be perfectly flexible yielding continuous Purchasing Power Parity (PPP). Second, it is assumed that money markets are always in equilibrium with respect to Keynesian Money Demand Function (KMDF) that defines real money balances as a function of real income and interest rate:

$M / P=L(Y, i)$

where $\mathrm{M}$ refers to the nominal money supply, $\mathrm{P}$ denotes the price level, $\mathrm{Y}$ is the real income and $\mathrm{i}$ is the interest rate.

According to KMDF, the demand for real money balances (M/P) is positively dependent on real income and negatively dependent on interest rate. It is acknowledged that a rise in real income induces higher level of real money demand to realise greater number of transactions. However, an increase in interest rate is expected to decrease the demand for real money balances due to the rise in cost of holding money. Consequently, $\mathrm{M}$ refers to the narrow definition of money supply, which is highly sensitive to interest rate.

Based on Cagan (1956) functional form, KMDF can be expressed with the following equation;

$M_{t} / P_{t}=Y_{t}^{k} e^{-\lambda i_{t}}$

where $\mathrm{k}$ and $\lambda$ represent the income elasticity and the interest semi-elasticity of money demand, respectively.

Taking natural logarithm in equation (2), we obtain ${ }^{2}$ :

\footnotetext{
${ }^{1}$ See Frenkel (1976), Mussa (1976) and Bilson (1978)

${ }^{2}$ In every following equation, lower-case letters are used to denote the variables in natural logarithm, except interest rate (i), which is always in level.
} 
$m_{t}-p_{t}=k y_{t}-\lambda i_{t}$

and solving equation (3) for $p$;

$p_{t}=m_{t}-k y_{t}+\lambda i_{t}$

For a foreign country the same equation applies:

$p_{t}^{*}=m_{t}^{*}-k^{*} y_{t}^{*}+\lambda * i_{t}^{*}$

where * denotes foreign variables and coefficients.

Intercountry difference renders:

$p_{t}-p_{t}^{*}=m_{t}-m_{t}^{*}-k y_{t}+k^{*} y_{t}{ }^{*}+\lambda i_{t}-\lambda * i_{t}^{*}$

Equation (6) states that relative prices are influenced by relative money supplies, real incomes and interest rates.

The second building block of FPMM is PPP. The law of one price, which states that identical products must sell for the same price, constitutes the foundation of PPP. According to the law of one price, arbitrage eliminates the discrepancy in prices. PPP extends the law of one price by incorporating price indices to the model and postulates that international trade removes arbitrage opportunities.

In its absolute version, PPP establishes a relationship between the exchange rate $\mathrm{S}$ (expressed as the domestic currency price of a unit of foreign currency) and the ratio of domestic and foreign prices ( $P$ and $P^{*}$, respectively), so that

$\mathrm{S}_{\mathrm{t}}=\mathrm{P}_{\mathrm{t}} / \mathrm{P}_{\mathrm{t}}^{*}$

or, taking natural logarithms;

$s_{t}=p_{t}-p_{t}^{*}$

Its implication is that an increase in the domestic price level or a decrease in the foreign price level results in depreciation of the exchange rate to retain purchasing power parity between domestic and foreign currencies.

Combining (8) and (6), we obtain FPMM;

$\mathrm{s}_{\mathrm{t}}=\mathrm{m}_{\mathrm{t}}-\mathrm{m}_{\mathrm{t}}^{*}-k \mathrm{y}_{\mathrm{t}}+\mathrm{k}^{*} \mathrm{y}_{\mathrm{t}} * \lambda \mathrm{i}_{\mathrm{t}}-\lambda \mathrm{i}_{\mathrm{t}} *$ 
Assuming that the domestic demand for money has identical elasticities to those of the demand for foreign currency (i. e., $\mathrm{k}=\mathrm{k}^{*}$ and $\lambda=\lambda^{*}$ ) provides the following restricted model;

$s_{t}=\left(m_{t}-m_{t}^{*}\right)-k\left(y_{t}-y_{t}^{*}\right)+\lambda\left(i_{t}-i_{t}^{*}\right)$

Equation (10) implies three testable hypotheses: (i) the coefficient on the relative money supply is positive and unity; (ii) the coefficient on the relative real income term is negative, and (iii) the coefficient on the relative interest rate is positive.

\subsection{Forward-Looking Monetary Model}

Flexible price monetary model can be modified to incorporate current expectations, denoted as $E_{t}$, by applying uncovered interest rate parity that states;

$E_{t}\left(s_{t+1}\right)-s_{t}=i_{t}-i_{t}^{*}$

Replacing interest rate differential in FPMM with the expected change in exchange rate provides;

$s_{t}=\left(m_{t}-m_{t}^{*}\right)-k\left(y_{t}-y_{t}^{*}\right)+\lambda\left[E_{t}\left(s_{t+1}\right)-s_{t}\right]$

Equation (12) can be rearranged as follows;

$\mathrm{s}_{\mathrm{t}}=(1+\lambda)^{-1} \mathrm{x}_{\mathrm{t}}+\delta\left[\mathrm{E}_{\mathrm{t}}\left(\mathrm{s}_{\mathrm{t}+1}\right)\right]$

where $x_{t}=\left[\left(m_{t}-m_{t}^{*}\right)-k\left(y_{t}-y_{t}^{*}\right)\right]$ and $\delta=\lambda(1+\lambda)^{-1}$

Based on rational expectations, the expected exchange rate in period $t+1$ can be written as:

$E_{t}\left(s_{t+1}\right)=(1+\lambda)^{-1} E_{t}\left(x_{t+1}\right)+\delta\left[E_{t}\left(s_{t+2}\right)\right]$

By replacing the expected exchange rate recursively for all future periods and imposing the transversality condition, $\lim _{j \rightarrow \infty} \delta^{j} \mathrm{E}_{\mathrm{t}}\left(\mathrm{s}_{\mathrm{t}+\mathrm{j}}\right)=0$, forward-looking monetary model (FLMM) can be defined with the equation below.

$\mathrm{S}_{\mathrm{t}}=(1+\lambda)^{-1} \sum_{j=0}^{\infty} \delta^{j} \mathrm{E}_{\mathrm{t}}\left(\mathrm{x}_{\mathrm{t}+\mathrm{j}}\right)$

$\delta$ can be considered as a discount factor. Accordingly, (15) states that exchange rate is the present value of all expected future values of $x_{t}$ (fundamentals); money supply and real income. However, future values of the fundamentals are not observable, thus FLMM is not practical to determine the exchange rate. Subtracting current value of the fundamentals from both sides of (15) makes the necessary 
arrangement that enables us to establish the link between exchange rate and fundamentals during the same period ${ }^{3}$.

$\mathrm{S}_{\mathrm{t}}-\mathrm{x}_{\mathrm{t}}=\sum_{j=1}^{\infty} \delta^{j} \mathrm{E}_{\mathrm{t}}\left(\Delta \mathrm{x}_{\mathrm{t}+\mathrm{j}}\right)$

If fundamentals are first-difference stationary then the right-hand side of (16) must be stationary. Consequently, the exchange rate is expected to be cointegrated with the fundamentals, provided that it is also first-difference stationary time series (see Macdonald and Taylor, 1992 for details). Thus, FLMM can be tested with the equation and constraints below by utilising a cointegration method, based on the assumption that there is no rational bubble;

$s_{t}=\beta_{0}\left(m_{t}-m_{t}^{*}\right)+\beta_{1}\left(y_{t}-y_{t}^{*}\right) \quad\left(\beta_{0}=1, \beta_{1}<0\right)$

\subsection{Real Interest Differential Model}

The Real Interest Differential Model (RIDM) was developed by Dornbusch (1976) and Frankel (1979). The model is based on the idea that prices are sticky and PPP holds only in the long-run so that inflation rate differentials can differ from interest rate differentials temporarily.

The model allows for short term deviations from PPP. Some well-known reasons for deviations from PPP are; transportation costs, tariffs and other legal barriers to commerce, non-tradable products and differences in the content of price indices across countries (see, e. g. Sosvilla-Rivero and García, 2006).

RIDM asserts that the initial rise in interest rate induces capital inflow and sharp appreciation of the domestic currency in the short-run, followed by a slow depreciation as prices adjust. That is due to rapid adjustment of asset prices while goods prices respond gradually, thus yielding the overshooting characteristic.

RIDM is based on three building block equations. The first one is uncovered interest rate parity expressed in (11). The second one postulates that FPMM determines only the long-run equilibrium exchange rate and replaces the interest rate differential in (10) with expected inflation rate differential;

$\bar{s}_{t}=\left(\bar{m}_{t}-\bar{m}_{t}^{*}\right)-k\left(\bar{y}_{t}-\bar{y}_{t}^{*}\right)+\lambda\left(\bar{\pi}_{t}-\bar{\pi}_{t}^{*}\right)$

where "bar" denotes the long-run equilibrium and $\pi$ refers to the expected inflation rate.

${ }^{3}$ We rewrite $x_{t}$ as; $x_{t}=(1+\lambda)^{-1} \sum_{j=0}^{\infty} \delta^{j} x_{t}$, and subtract it from the right-hand side of Equation (15) to obtain Equation (16). 
The third equation states that the exchange rate is expected to change at the rate of expected inflation differential when the long-run equilibrium is obtained for s;

$\mathrm{E}_{\mathrm{t}}\left(s_{t+1}\right)-s_{t}=\theta\left(\bar{s}_{t}-s_{t}\right)+\left(\bar{\pi}_{t}-\bar{\pi}_{t}^{*}\right)$

where $\theta$ is the speed of adjustment, which depends on the degree of price stickiness, towards the equilibrium level.

Solving for $s_{t}$ by using (11), (18) and (19) provides;

$s_{t}=\left(\bar{m}_{t}-\bar{m}_{t}{ }^{*}\right)-k\left(\bar{y}_{t}-\bar{y}_{t}{ }^{*}\right)-(1 / \theta)\left(i_{t}-i_{t}{ }^{*}\right)+(\lambda+1 / \theta)\left(\bar{\pi}_{t}-\bar{\pi}_{t}{ }^{*}\right)$

RIDM is obtained by rewriting (20) as below:

$s_{t}=\left(\bar{m}_{t}-\bar{m}_{t}^{*}\right)-k\left(\bar{y}_{t}-\bar{y}_{t}^{*}\right)-(1 / \theta)\left(r_{t}-r_{t}^{*}\right)+\lambda\left(\bar{\pi}_{t}-\bar{\pi}_{t}^{*}\right)$

where $r$ is the real interest rate

On assuming that short interest rates capture real interest rates (i.e. the liquidity impulses of monetary policy) and long bond yields capture expected inflation (MacDonald, 2007), RIDM can be tested based on the equation and constraints below (for further details see Frankel, 1979 or MacDonald, 2007, p:137);

$s_{t}=\alpha_{0}\left(m_{t}-m_{t}^{*}\right)+\alpha_{1}\left(y_{t}-y_{t}^{*}\right)+\alpha_{2}\left(i_{t}^{S}-i_{t}^{S *}\right)+\alpha_{3}\left(i_{t}^{L}-i_{t}^{L *}\right) \quad\left(\alpha_{0}=1 ; \alpha_{1}, \alpha_{2}<0 ; \alpha_{3}>0\right)$

where $i^{S}$ and $i^{L}$ denote, respectively, short term and long term interest rates

FPMM postulates that real interest rates are always equal to each other across countries due to international capital movements seeking excess profit opportunity, whereas RIDM allows for temporary differences in real interest rates. Thus, RIDM is not considered as a long-run model, since it incorporates short term influences. Nevertheless, it is utilised in some researches to test the monetary model for the long-run (see, e.g. Baillie and Selover, 1987; Kearney and MacDonald, 1990; Cheung and Chinn, 1998; Ketenci and Uz, 2008; Hunter and Menla Ali, 2014)

\subsection{Possible reasons for the failure of the monetary models}

Since its conception in the 1970s, the monetary model has become the dominant theoretical model of exchange rate determination. Researchers test FPMM and its variants by employing variety of currency parities, econometric techniques and research methodologies. In general, the monetary models perform very poorly and empirical findings do not support the expected link between macro fundamentals and exchange rates based on country-by-country analysis during the floating exchange rate period, while panel data estimates provide mixed but more support for the models (see, e. g. Husted and MacDonald, 1998; Groen, 2000; Rapach and Wohar, 2004; Ketenci and Uz, 2008; Cerra and Saxena, 2010). 
Regarding the (absolute) PPP assumption, a building block of the monetary model that relates exchange rates to prices, it is almost always rejected (see, e. g. Sosvilla-Rivero and García, 2006). For that reason, there is a common belief that the empirical failure of the monetary models is mainly due to deviations from PPP. As Smith and Wickens (1984) state: “...empirical tests of the exchange rate equation arising from the monetary model very often lead to rejection of the model. The blame for this is usually attributed to the breakdown of the purchasing power parity assumption."

Thus, the extended versions incorporate additional components into FPMM to allow for deviations from PPP. RIDM is the case, which attends to short term deviations. Another version, known as HooperMorton model, enriches FPMM further with cumulative trade balance to allow for long term deviations from PPP (see Hooper and Morton, 1982 for details), whereas an alternative version adds the relative price of "tradables to nontradables" into RIDM, based on the assumption that PPP holds only for tradable products in the long-run (see Chinn and Meese, 1995 for an overview of the extended monetary models).

Although academia has devoted considerable attention to deviations from PPP; we assert that the failure of monetary models is also due to insufficiency of Keynesian money demand function (KMDF) to formulate all the motivations for money demand. KMDF neglects intercountry money demand. Consequently, failure of the monetary models, especially for the currency parities comprising "reserve currencies" that are commonly used in international transactions, is not surprising for us.

We contend that the most of the empirical studies on the monetary models employ currency parities that encompass commonly traded currencies such as the US Dollar, the Euro, the Sterling Pound or the Japanese Yen. However, these currencies are "reserve currencies" 4 , meaning that they are demanded and kept in reserves by many central banks in the world to be able to maintain financial stability and to intervene in the FX market when necessary. There is global demand for those currencies, especially for the US Dollar and the Euro, since much of the amount of international trade and direct investment settled in these currencies. In addition, there are large volumes of both assets and currency swap agreements denominated in these currencies.

Nevertheless, the implicit assumption of the monetary models is that currencies are not internationally demanded as reserve currencies. The narrow perception, which ignores several reasons to demand foreign currencies, is one of the important shortcomings of the models. Currency substitution is the case of demanding a reserve currency in parallel to or instead of the domestic currency and a crucial situation that violates the mechanism of monetary models. Consider the case that domestic currency is not a reserve currency but the foreign currency is. According to the monetary models, nominal exchange rate is supposed to appreciate when real income in the home country increases. However, in reality,

\footnotetext{
${ }^{4}$ Three sets of factors have been identified as critical for producing an international currency: (i) economic weights, (ii) openness and depth of financial markets, (iii) and credibility of economic and legal systems (see, e. g., Eichengreen, 2005, and Helleiner, 2008).
} 
appreciation of the domestic currency may not occur as expected because it is not possible to detach the demand for the domestic currency from that for the foreign (reserve) currency.

The shortcomings of monetary models are not limited to the deviations from PPP and to insufficient specification of money demand function. Monetary models decompose real money balances (M/P) and use money supply and price level individually to incorporate PPP. However, this method imposes a restriction for the econometric model that requires one-to-one relationship between price level and money supply. Besides, the model postulates that income elasticity and interest semi-elasticity of money demand are stable. Yet, it is very likely that they have been changing due to financial crises, regulatory changes that occurred in the banking sector and development of financial systems (e. g. using credit cards instead of money) (see, e. g. Huynh and Schmidt-Dengler, 2014, and Lucas and Nicolini, 2015).

We claim that monetary models are fundamentally flawed due to the several reasons explained above and some supportive results for the models based on panel data estimations are spurious, since varieties in economic structures of countries (in terms of some criteria such as; international demand for the currency, being net oil importer/exporter, having steady current account surplus/deficit) are mainly ignored.

\section{Data and Methodology}

\subsection{Data}

The analysis covers the Organisation for Economic Co-operation and Development (OECD) member countries ${ }^{5}$. Their currencies are used to form several currency pairs, which can be considered as 2 groups;

1) The pairs that contain a highly traded reserve currency; the US Dollar, the Euro, the British Pound, the Japanese Yen, the Australian Dollar, the Canadian Dollar and the Swiss Frank.

2) The pairs that consist of currencies, which are not major global reserve currencies; the Mexican Peso, the Swedish Krona, the Norwegian Krone, the Polish Zloty, the Czech Koruna, the South Korean Won, the Israeli Shekel, the Hungarian Forint, the Icelandic Krona and the Turkish Lira.

The first group is employed to observe the effects of reserve currencies on the results. As it is explained, monetary models are incapable of formulating the demand for reserve currencies. Thus, the monetary models are expected to perform poorly especially for the pairs that consist of the US Dollar, which is the

\footnotetext{
${ }^{5}$ The OECD member countries; Austria, Belgium, Estonia, Finland, France, Germany, Greece, Ireland, Italy, Luxembourg, the Netherlands, Portugal, Slovakia, Slovenia and Spain are treated as Euro Area in the research. The analysis covers the remaining OECD members individually: Australia, Canada, Chile, Czech Rebuplic, Denmark, Hungary, Iceland, Israel, Japan, S. Korea, Mexico, New Zealand, Norway, Poland, Sweden, Switzerland, Turkey, the UK and the USA.
} 
most dominant reserve currency, and a currency for which international demand is negligible. Nevertheless, the situation is much more complicated for the pairs that comprise two reserve currencies.

The second group is used for the appropriate test of the models, meaning that, since international demand for those currencies are lower; it is more likely that monetary models yield the expected results, provided that PPP and KMDF hold. Employing the second group currency pairs enables us to test the models in an unbiased way.

For each country except Denmark, which conducts fixed exchange rate policy, data set covers free floating period. Managed floating period is mainly excluded from the analysis because theoretical foundation of the monetary model depends on the free-market economy; supply and demand mechanism is applied to currency markets. Denmark is not dropped from the research to address all the OECD member countries and to see whether the results are affected by the exchange rate system.

The data is mainly obtained from OECD and central banks. M1 is chosen as the relevant money supply $(\mathrm{M})$ to be in line with the theory explained in the section 2.1. Real income $(\mathrm{Y})$ and price level $(\mathrm{P})$ are proxied by industrial production and consumer price indices, while $i^{L}$ and $i^{S}$ denote long term and short term interest rates, respectively.

M1, industrial production and consumer price indices are seasonally adjusted. They are in natural logarithmic form as well as exchange rates, whereas interest rates are in levels. Availability of data and inception of free-floating exchange rate system determine the research period for each country pair. Besides, structural breaks created by adoption of inflation targeting monetary policy are taken into account. Further information about the data set is provided in Appendix-A.

\subsection{Methodology}

To carry out the analysis, Johansen cointegration technique (Johansen, 1988; Johansen and Juselius, 1990 ) is employed. The method requires using time series, which are stationary in their first-differenced form.

A time series is said to be stationary, if its mean, variance and autocovariance (at various lags) remain the same no matter at what point we measure them; that is, they are time invariant (Gujarati and Porter, 2009). If a time series has to be differenced $d$ times to become stationary, then it is denoted as $I(d)$; integrated order $d$. Stationarity is examined by unit root tests. Two popular unit-root tests are the Augmented Dickey-Fuller (ADF) and the Phillips-Perron (PP) tests.

The null hypothesis of ADF test is $\delta=0$ in the following equation, where $t$ denotes trend; 
$\Delta \mathrm{Y}_{\mathrm{t}}=\beta_{1}+\beta_{2} t+\delta \mathrm{Y}_{\mathrm{t}-1}+\sum_{i=1}^{m} \alpha_{\mathrm{i}} \Delta \mathrm{Y}_{\mathrm{t}-\mathrm{i}}+\varepsilon_{\mathrm{t}}$

Accepting the null hypothesis indicates that the series is not stationary. An alternative test is introduced by Phillips (1987), Phillips and Perron (1988). Rather than including extra lags of $\Delta y_{t}$ (as in ADF test) to cope with autocorrelation, they suggest allowing for weak dependence and heterogenity in $\varepsilon_{\mathrm{t}}$ by using nonparametric statistical methods.

Cointegration techniques are used to identify long-run economic relationships among non-stationary variables. A set of $I(1)$ variables is called cointegrated if a linear combination exists, which is $I(0)$. Johansen cointegration is the multivariate case. Hwang (2001) summarises this method as follows;

Let $X_{t}$ be $(n \times 1)$ vector $I(1)$ variables and assume this vector has a $k$ th order vector autoregressive (VAR) representation with Gaussian errors, $\varepsilon_{\mathrm{t}}$ :

$X_{t}=A_{1} X_{t-1}+\ldots+A_{k} X_{t-k}+\varepsilon_{t}$

where each of $A_{i}$ is $(n \times n)$ matrix of parameters. Letting $\Delta$ represent the first-difference operator, (24) can be reformulated into a vector error correction form:

$\Delta \mathrm{X}_{\mathrm{t}}=\Gamma_{1} \Delta \mathrm{X}_{\mathrm{t}-1}+\ldots+\Gamma_{\mathrm{k}} \Delta \mathrm{X}_{\mathrm{t}-\mathrm{k}+1}+\Pi \mathrm{X}_{\mathrm{t}-\mathrm{k}}+\varepsilon_{\mathrm{t}}$

where;

$\Gamma_{i}=-\left(I-A_{1}-\ldots-A_{i}\right), \quad(i=1, \ldots, k-1)$

$\Pi=-\left(I-A_{1}-\ldots-A_{k}\right)$

Cointegration can be detected by examining the $(n \times n) \Pi$ matrix $\left(\Pi=\alpha \beta^{\prime}\right)$, where $\alpha$ represents the speed of adjustment to disequilibrium and $\beta$ is a matrix of long-run coefficients. The likelihood ratio; or Trace, test statistic is commonly used to determine the rank $(r)$ of $\Pi$. The test starts with the null hypothesis $r=0$ (no cointegration) against the alternative $r \leq 1$ (at least one cointegrating vector) and subsequent further orders of cointegration $r=i$ against the alternative $r \leq i+1$; the sequence stops at $r=i$ when the null cannot be rejected (Hunter and Menla Ali, 2014). Trace test statistics can be expressed in terms of eigenvalues $\left(\lambda_{\mathrm{i}}\right)$ and sample size $(T)$;

$\lambda_{\text {trace }}=-T \sum_{i=r+1}^{n}\left(1-\lambda_{i}\right)$ 


\section{Empirical Analysis}

To apply Johansen cointegration, first, integration order of variables is examined by Augmented Dickey Fuller (ADF) and Philips Perron (PP) tests. For some series, unit root test results do not match. For those series, we rely on PP test results and conclude that all series are $I(1)$. Johansen cointegration is applied to FPMM, FLMM, RIDM, PPP and KMDF. Unit root and Trace test results are presented in Appendix-B and Appendix-C, respectively. Significance level for both of the tests is $5 \%$.

Table-1, Table-2 and Table-3 provide cointegration results for the three variants of the monetary model with unrestricted coefficients. We prefer not to utilise the models with restricted coefficients since probable failure of the models could be attributed to the assumption of identical elasticities for the variables across countries, which is applied by restricting the coefficients.

Lag levels for vector error correction models are specified based on Akaike information criterion. The numbers in columns headed $m, m^{*}, y, y^{*}, i^{S}, i^{S *}, i^{L}, i^{L *}$ are the coefficient estimates of the variables. Standard errors are given in parentheses. In every table, country pairs are marked with *** when estimation provides the expected results with entirely significant coefficients and * is used when there are some insignificant coefficients, although all coefficient signs are in line with the theoretical model. Coefficients are considered as significant if they (in absolute value) are at least twice the standard errors.

The expected results for each monetary model version are as follows;

FPMM: significantly positive coefficients for $m, y^{*}, i^{L}$; significantly negative coefficients for $m^{*}, y, i^{L *}$ FLMM: significantly positive coefficients for $m, y^{*}$; significantly negative coefficients for $m^{*}, y$ RIDM: significantly positive coefficients for $m, y^{*}, i^{S_{*}}, i^{L}$; significantly negative coefficients for $m^{*}, y, i^{S}, i^{L *}$

Although the theory requires unity coefficient for money supply, we do not focus on the magnitude. That is because the unity coefficient depends on two conditions; absolute PPP and one-to-one relationship between price level and money supply, which is the outcome of decomposing real money balances (see equations; 3 and 4). Both of the conditions must hold strictly to obtain unity coefficient for money supply. We allow for flexibility in the coefficient of money supply since those conditions are extremely binding.

According to the results in the tables, FPMM always fails to establish the expected links between macroeconomic variables and exchange rates. FLMM provides favourable results only for Sweden-USA and UK-Euro Area. RIDM provides the expected coefficient signs for Euro Area-USA and Mexico-USA. However, $m$ is not statistically significant in the case of Euro Area-USA. 


\section{TABLE-1}

Cointegration Results: Flexible Price Monetary Model

\begin{tabular}{|c|c|c|c|c|c|c|}
\hline Home-Foreign & $m$ & $m^{*}$ & $y$ & $y^{*}$ & $i^{L}$ & $i^{L *}$ \\
\hline Australia-USA & $-2.48(0.94)$ & $-1.44(1.10)$ & $3.56(3.64)$ & $-2.82(1.98)$ & $-0.23(0.08)$ & $-0.66(0.11)$ \\
\hline Canada-USA & $-1.02(0.10)$ & $0.99(0.14)$ & $-0.41(0.41)$ & $2.41(0.46)$ & $0.16(0.03)$ & $-0.07(0.03)$ \\
\hline Chile-USA & $-0.21(0.08)$ & $0.58(0.16)$ & $-1.02(0.30)$ & $-0.19(0.30)$ & $-0.04(0.02)$ & $0.05(0.02)$ \\
\hline Czech R.-USA & $-1.91(0.24)$ & $2.01(0.24)$ & $2.23(0.51)$ & $-3.74(0.69)$ & $-0.06(0.02)$ & $0.04(0.02)$ \\
\hline Denmark-USA & $-1.10(0.53)$ & $-0.11(0.85)$ & $5.92(2.41)$ & $-12.8(2.84)$ & $-0.73(0.19)$ & $-0.23(0.21)$ \\
\hline Euro Area-USA & $-1.57(0.30)$ & $3.74(0.56)$ & $13.2(2.04)$ & $-13.7(2.17)$ & $-0.20(0.08)$ & $0.11(0.06)$ \\
\hline Hungary-USA & $2.69(1.24)$ & $-0.26(0.85)$ & $4.94(1.45)$ & $-13.3(2.62)$ & $-0.24(0.05)$ & $-0.43(0.12)$ \\
\hline Iceland-USA & $0.41(0.22)$ & $-1.01(0.88)$ & $0.22(0.32)$ & $-5.65(2.28)$ & $-0.13(0.03)$ & $0.46(0.08)$ \\
\hline Israel-USA & $-1.48(0.21)$ & $1.38(0.25)$ & $0.22(0.43)$ & $-3.93(0.64)$ & $-0.27(0.04)$ & $0.01(0.03)$ \\
\hline Japan-USA & $-1.84(1.68)$ & $0.26(0.64)$ & $-0.02(0.69)$ & $0.35(1.24)$ & $-1.02(0.16)$ & $0.27(0.06)$ \\
\hline S.Korea-USA & $-0.03(0.06)$ & $0.40(0.05)$ & $-0.35(0.05)$ & $-1.42(0.11)$ & $0.03(0.01)$ & $-0.03(0.01)$ \\
\hline Mexico-USA & $-8.28(2.09)$ & $11.5(2.31)$ & $37.1(11.2)$ & $-22.2(6.45)$ & $-0.13(0.14)$ & $1.45(0.26)$ \\
\hline New Zealand-USA & $-7.74(1.26)$ & $2.86(1.28)$ & $18.8(3.69)$ & $14.9(2.96)$ & $0.78(0.17)$ & $-0.02(0.32)$ \\
\hline Norway-USA & $-0.61(0.09)$ & $0.48(0.20)$ & $-1.35(0.50)$ & $-1.45(0.35)$ & $0.09(0.03)$ & $-0.04(0.03)$ \\
\hline Poland-USA & $-2.27(0.24)$ & $1.48(0.15)$ & $4.01(0.58)$ & $-4.83(0.52)$ & $-0.11(0.02)$ & $0.12(0.03)$ \\
\hline Sweden-USA & $-2.42(0.99)$ & $7.82(1.97)$ & $10.6(3.26)$ & $-18.6(4.53)$ & $0.72(0.19)$ & $-0.24(0.21)$ \\
\hline Switzerland-USA & $-1.03(2.00)$ & $0.80(2.02)$ & $5.23(3.19)$ & $13.5(2.56)$ & $0.18(0.28)$ & $1.69(0.29)$ \\
\hline Turkey-USA & $0.62(0.24)$ & $0.22(0.34)$ & $-2.45(0.44)$ & $0.79(0.56)$ & $0.03(0.01)$ & $-0.09(0.02)$ \\
\hline UK-USA & $0.15(0.12)$ & $1.28(0.25)$ & $5.99(1.25)$ & $-5.43(0.80)$ & $-0.31(0.04)$ & $0.12(0.04)$ \\
\hline Japan-Euro Area & $-1.02(5.19)$ & $5.15(1.55)$ & $20.4(3.89)$ & $-21.9(5.96)$ & $1.41(0.53)$ & $0.92(0.33)$ \\
\hline UK-Euro Area & $-0.88(0.87)$ & $5.26(1.07)$ & $25.1(3.11)$ & $-14.6(1.67)$ & $-0.48(0.09)$ & $0.42(0.09)$ \\
\hline Hungary-Czech R. & $-1.09(0.25)$ & $1.72(0.27)$ & $5.40(0.47)$ & $-5.44(0.52)$ & $0.08(0.01)$ & $0.06(0.03)$ \\
\hline S.Korea-Czech R. & $-0.33(0.27)$ & $0.28(0.15)$ & $1.16(0.31)$ & $-1.09(0.30)$ & $0.09(0.03)$ & $0.05(0.02)$ \\
\hline Mexico-Czech R. & $11.6(2.64)$ & $-17.5(3.79)$ & $-40.8(5.83)$ & $18.7(2.41)$ & $-0.28(0.11)$ & $-0.65(0.16)$ \\
\hline Norway-Czech R. & $-2.88(0.99)$ & $5.35(0.92)$ & $9.97(1.55)$ & $-0.39(0.86)$ & $0.09(0.09)$ & $0.13(0.07)$ \\
\hline Poland-Czech R. & $0.66(0.44)$ & $-2.92(0.63)$ & $5.74(0.69)$ & $-3.99(0.44)$ & $-0.07(0.03)$ & $0.01(0.04)$ \\
\hline Sweden-Czech R. & $5.33(1.05)$ & $-0.84(0.74)$ & $7.78(0.76)$ & $-7.74(0.76)$ & $-0.01(0.05)$ & $0.15(0.03)$ \\
\hline Norway-Hungary & $-3.96(2.63)$ & $2.24(0.95)$ & $-2.14(1.34)$ & $-1.21(0.83)$ & $0.01(0.10)$ & $-0.16(0.05)$ \\
\hline Norway-Iceland & $0.10(0.38)$ & $0.37(0.13)$ & $2.44(0.93)$ & $-0.94(0.26)$ & $0.05(0.02)$ & $-0.01(0.01)$ \\
\hline Norway-Israel & $0.34(0.43)$ & $-1.30(0.30)$ & $0.21(0.21)$ & $0.49(0.20)$ & $0.19(0.02)$ & $-0.26(0.04)$ \\
\hline Norway-S.Korea & $1.73(1.75)$ & $-5.50(2.30)$ & $-37.2(4.28)$ & $-10.6(3.30)$ & $-1.02(0.35)$ & $1.09(0.34)$ \\
\hline Norway-Mexico & $-1.10(0.15)$ & $0.83(0.17)$ & $1.10(0.37)$ & $1.24(0.43)$ & $0.15(0.02)$ & $0.01(0.01)$ \\
\hline Norway-Poland & $-1.96(0.55)$ & $1.95(0.35)$ & $5.74(0.71)$ & $1.02(0.63)$ & $0.05(0.04)$ & $0.04(0.03)$ \\
\hline Norway-Turkey & $1.62(0.36)$ & $-0.49(0.15)$ & $1.73(0.57)$ & $-0.42(0.26)$ & $0.04(0.03)$ & $-0.03(0.01)$ \\
\hline Sweden-Hungary & $-0.41(0.29)$ & $1.13(0.21)$ & $2.93(0.35)$ & $-3.84(0.37)$ & $0.07(0.02)$ & $-0.03(0.01)$ \\
\hline Sweden-Iceland & \multicolumn{6}{|c|}{ No Cointegration } \\
\hline Sweden-S.Korea & $0.61(0.29)$ & $-0.07(0.23)$ & $0.59(0.32)$ & $-1.66(0.29)$ & $-0.01(0.03)$ & $-0.08(0.04)$ \\
\hline Sweden-Mexico & $2.46(0.59)$ & $-3.66(0.55)$ & $-5.44(0.65)$ & $12.9(1.60)$ & $-0.02(0.04)$ & $0.02(0.02)$ \\
\hline Sweden-Poland & $17.5(3.57)$ & $-3.41(1.63)$ & $9.27(1.55)$ & $-13.1(2.25)$ & $-0.27(0.10)$ & $0.41(0.09)$ \\
\hline Sweden-Turkey & $-9.07(2.96)$ & $4.86(1.81)$ & $9.11(3.64)$ & $-4.31(3.79)$ & $0.65(0.18)$ & $-0.15(0.04)$ \\
\hline
\end{tabular}

Note: Standard errors are given in parentheses. 
TABLE-2

Cointegration Results: Forward-Looking Monetary Model

\begin{tabular}{|c|c|c|c|c|}
\hline Home-Foreign & $m$ & $m *$ & $y$ & $y^{*}$ \\
\hline Australia-USA & \multicolumn{4}{|c|}{ No Cointegration } \\
\hline Canada-USA & $1.45(0.31)$ & $-2.12(0.44)$ & $3.84(1.22)$ & $-3.01(1.17)$ \\
\hline Chile-USA & $-0.62(0.24)$ & $-0.05(0.31)$ & $0.97(0.93)$ & $1.44(1.09)$ \\
\hline Czech R.-USA & $-1.86(0.26)$ & $2.01(0.26)$ & $1.99(0.54)$ & $-2.87(0.63)$ \\
\hline Denmark-USA & $-1.97(2.73)$ & $-7.25(3.91)$ & $-71.9(12.0)$ & $54.1(11.5)$ \\
\hline Euro Area-USA & $-6.49(2.02)$ & $20.1(4.12)$ & $69.6(16.0)$ & $-73.1(15.0)$ \\
\hline Hungary-USA & $-0.69(0.50)$ & $1.78(0.26)$ & $2.90(0.79)$ & $-5.66(1.31)$ \\
\hline Iceland-USA & $-0.09(0.07)$ & $-1.52(0.30)$ & $1.08(0.13)$ & $-4.26(0.87)$ \\
\hline Israel-USA & $-0.82(0.13)$ & $0.78(0.15)$ & $0.79(0.30)$ & $-1.76(0.40)$ \\
\hline Japan-USA & $-0.04(1.08)$ & $-0.03(0.35)$ & $-0.77(0.63)$ & $1.64(0.99)$ \\
\hline S. Korea-USA & $-9.58(21.1)$ & $-101(21.3)$ & $41.7(31.9)$ & $313(68.3)$ \\
\hline Mexico-USA & $-0.23(0.22)$ & $0.53(0.25)$ & $-0.26(1.71)$ & $-0.20(1.12)$ \\
\hline New Zealand-USA & $-0.85(0.20)$ & $-0.68(0.20)$ & $1.75(0.80)$ & $1.91(0.46)$ \\
\hline Norway-USA & $4.82(1.09)$ & $4.21(1.92)$ & $44.5(7.91)$ & $6.54(3.75)$ \\
\hline Poland-USA & $-1.51(0.26)$ & $1.06(0.11)$ & $2.36(0.59)$ & $-2.28(0.42)$ \\
\hline Sweden-USA $* * *$ & $9.17(4.07)$ & $-24.5(5.69)$ & $-37.7(11.8)$ & $68.9(15.7)$ \\
\hline Switzerland-USA & $-0.74(0.21)$ & $-0.39(0.22)$ & $-0.03(0.45)$ & $1.91(0.31)$ \\
\hline Turkey-USA & $-0.002(0.19)$ & $1.06(0.24)$ & $-2.33(0.41)$ & $2.05(0.42)$ \\
\hline UK-USA & $0.08(0.28)$ & $1.09(0.64)$ & $8.53(2.88)$ & $-2.08(1.57)$ \\
\hline Japan-Euro Area & $-27.7(6.59)$ & $11.7(2.37)$ & $34.5(5.81)$ & $-29.9(8.19)$ \\
\hline UK-Euro Area $^{* * *}$ & $13.1(2.94)$ & $-22.9(3.58)$ & $-51.3(8.47)$ & $33.2(5.70)$ \\
\hline$\overline{\text { Hungary-Czech R. }}$ & $0.33(0.24)$ & $0.09(0.17)$ & $-3.91(0.42)$ & $4.27(0.47)$ \\
\hline S.Korea-Czech R. & $-1.59(0.24)$ & $0.54(0.22)$ & $1.33(0.42)$ & $-1.06(0.37)$ \\
\hline Mexico-Czech R. & $-5.34(2.26)$ & $4.35(2.98)$ & $23.2(4.10)$ & $-10.6(1.85)$ \\
\hline Norway-Czech R. & $1.57(0.30)$ & $-1.48(0.25)$ & $-2.53(0.48)$ & $-0.58(0.29)$ \\
\hline Poland-Czech R. & $0.27(0.19)$ & $-1.71(0.32)$ & $4.25(0.49)$ & $-3.06(0.31)$ \\
\hline Sweden-Czech R. & $-63.5(16.9)$ & $10.0(11.4)$ & $-111(12.1)$ & $109(11.8)$ \\
\hline Norway-Hungary & $-5.20(1.00)$ & $1.99(0.48)$ & $-2.68(0.63)$ & $0.24(0.40)$ \\
\hline Norway-Iceland & 8.55 (3.69) & $0.63(1.35)$ & $61.0(8.59)$ & $-5.36(2.52)$ \\
\hline Norway-Israel & $-83.5(13.3)$ & $20.5(3.94)$ & $50.0(7.61)$ & $4.85(7.28)$ \\
\hline Norway-S.Korea & $1.12(0.91)$ & $-3.43(0.94)$ & $-16.3(2.16)$ & $-3.86(1.69)$ \\
\hline Norway-Mexico & $-1.95(0.89)$ & $3.78(0.89)$ & $14.2(2.50)$ & $-1.42(2.79)$ \\
\hline Norway-Poland & $-2.23(0.59)$ & $2.15(0.42)$ & $6.92(0.86)$ & $0.96(0.74)$ \\
\hline Norway-Turkey & $2.70(0.47)$ & $-0.56(0.17)$ & $0.62(0.79)$ & $-0.75(0.37)$ \\
\hline Sweden-Hungary & $-1.86(0.41)$ & $-2.22(0.45)$ & $-6.94(0.78)$ & $7.04(0.81)$ \\
\hline Sweden-Iceland & $-1.93(1.94)$ & $-1.25(0.42)$ & $17.9(3.07)$ & $-0.47(0.65)$ \\
\hline Sweden-S.Korea & $0.50(0.26)$ & $0.57(0.16)$ & $0.82(0.24)$ & $-1.64(0.27)$ \\
\hline Sweden-Mexico & \multicolumn{4}{|c|}{ No Cointegration } \\
\hline Sweden-Poland & $-11.6(2.15)$ & $5.61(1.01)$ & $-2.01(1.02)$ & $2.19(1.66)$ \\
\hline Sweden-Turkey & $1.17(0.49)$ & $0.26(0.26)$ & $2.93(0.59)$ & $-3.61(0.64)$ \\
\hline
\end{tabular}

Note: Standard errors are given in parentheses. 


\section{TABLE-3}

Cointegration Results: Real Interest Differential Model

\begin{tabular}{|c|c|c|c|c|c|c|c|c|}
\hline Home-Foreign & $m$ & $m *$ & $\boldsymbol{y}$ & $y^{*}$ & $i^{S}$ & $i^{S *}$ & $i^{L}$ & $i^{L *}$ \\
\hline Australia-USA & $-1.74(0.25)$ & $-2.23(0.29)$ & $5.43(0.96)$ & $2.77(0.56)$ & $-0.24(0.02)$ & $0.07(0.02)$ & $0.40(0.04)$ & $-0.42(0.04)$ \\
\hline Canada-USA & $-0.94(0.11)$ & $0.86(0.14)$ & $-0.70(0.38)$ & $2.64(0.44)$ & $-0.02(0.02)$ & $-0.02(0.02)$ & $0.19(0.04)$ & $-0.05(0.04)$ \\
\hline Chile-USA & $-0.32(0.07)$ & $0.29(0.15)$ & $0.40(0.36)$ & $1.26(0.33)$ & $-0.05(0.01)$ & $-0.01(0.01)$ & $0.06(0.02)$ & $-0.02(0.02)$ \\
\hline Czech R.-USA & $-1.48(0.26)$ & $1.34(0.34)$ & $1.14(0.54)$ & $-1.59(0.85)$ & $-0.15(0.03)$ & $0.03(0.01)$ & $0.06(0.03)$ & $-0.02(0.02)$ \\
\hline Denmark-USA & $-0.72(0.17)$ & $0.73(0.26)$ & $2.96(0.65)$ & $-3.67(0.77)$ & $0.01(0.01)$ & $-0.07(0.03)$ & $-0.53(0.05)$ & $0.56(0.07)$ \\
\hline Euro Area-USA ${ }^{*}$ & $0.20(0.32)$ & $-2.61(0.72)$ & $-7.06(1.98)$ & $8.90(2.42)$ & $-0.46(0.06)$ & $0.15(0.04)$ & $0.48(0.10)$ & $-0.23(0.07)$ \\
\hline Hungary-USA & $0.66(0.40)$ & $-2.05(0.36)$ & $-5.36(0.56)$ & $11.65(1.29)$ & $0.10(0.02)$ & $-0.60(0.09)$ & $0.02(0.02)$ & $0.005(0.03)$ \\
\hline Iceland-USA & $4.53(1.02)$ & $-28.6(4.46)$ & $-0.11(1.41)$ & $-35.9(12.6)$ & $-0.12(0.09)$ & $0.40(0.17)$ & $0.14(0.13)$ & $-0.77(0.32)$ \\
\hline Israel-USA & $-1.58(0.25)$ & $1.68(0.25)$ & $0.51(0.41)$ & $-5.08(0.67)$ & $0.06(0.02)$ & $-0.01(0.03)$ & $-0.33(0.06)$ & $0.05(0.03)$ \\
\hline Japan-USA & $-6.02(4.11)$ & $3.92(1.60)$ & $0.50(1.13)$ & $-2.44(2.27)$ & $-1.79(0.26)$ & $0.15(0.07)$ & $0.48(0.27)$ & $0.25(0.12)$ \\
\hline S. Korea-USA & $-0.24(0.07)$ & $0.44(0.05)$ & $-0.25(0.04)$ & $-1.11(0.17)$ & $-0.01(0.01)$ & $-0.01(0.01)$ & $0.04(0.01)$ & $-0.01(0.01)$ \\
\hline Mexico-USA ${ }^{* * *}$ & $2.09(0.30)$ & $-2.50(0.37)$ & $-9.70(1.60)$ & $5.21(0.96)$ & $-0.16(0.04)$ & $0.07(0.02)$ & $0.12(0.04)$ & $-0.31(0.05)$ \\
\hline New Zealand-USA & $10.4(1.59)$ & $-2.39(1.63)$ & $-41.8(5.86)$ & $-5.68(4.30)$ & $0.37(0.15)$ & $-0.93(0.21)$ & $-1.31(0.28)$ & $1.43(0.44)$ \\
\hline Norway-USA & $-0.57(0.06)$ & $0.69(0.13)$ & $-0.21(0.30)$ & $-1.65(0.30)$ & $-0.03(0.01)$ & $0.05(0.01)$ & $0.11(0.04)$ & $-0.07(0.04)$ \\
\hline Poland-USA & $-0.09(0.31)$ & $-0.37(0.24)$ & $-2.23(0.77)$ & $1.83(0.77)$ & $-0.29(0.03)$ & $0.04(0.02)$ & $0.20(0.05)$ & $-0.45(0.05)$ \\
\hline Sweden-USA & $-1.39(0.85)$ & $5.45(1.73)$ & $6.95(2.83)$ & $-12.6(4.22)$ & $-0.67(0.13)$ & $0.84(0.13)$ & $2.49(0.33)$ & $-2.42(0.32)$ \\
\hline Switzerland-USA & $16.4(6.77)$ & $-9.25(6.38)$ & $-15.9(11.2)$ & $-61.8(9.48)$ & $-2.71(0.75)$ & $1.33(0.67)$ & $3.98(1.53)$ & $-6.21(1.14)$ \\
\hline Turkey-USA & $1.12(0.16)$ & $-0.19(0.22)$ & $-1.32(0.22)$ & $-0.95(0.31)$ & $0.06(0.01)$ & $0.06(0.01)$ & $-0.03(0.01)$ & $-0.05(0.01)$ \\
\hline UK-USA & $-0.39(0.05)$ & $-0.11(0.11)$ & $-0.74(0.48)$ & $0.94(0.36)$ & $-0.09(0.01)$ & $0.01(0.01)$ & $-0.01(0.02)$ & $0.06(0.02)$ \\
\hline Japan-Euro Area & $2.31(0.63)$ & $-0.34(0.29)$ & $1.49(0.34)$ & $-7.44(0.73)$ & $0.15(0.15)$ & $0.26(0.04)$ & $-0.16(0.05)$ & $0.003(0.03)$ \\
\hline UK-Euro Area & $-0.08(0.96)$ & $-3.67(1.19)$ & $-27.4(3.33)$ & $16.7(2.05)$ & $0.05(0.07)$ & $-0.14(0.09)$ & $0.72(0.10)$ & $-0.52(0.11)$ \\
\hline Hungary-Czech R. & $-0.97(0.17)$ & $1.87(0.28)$ & $-2.34(0.54)$ & $0.80(0.55)$ & $-0.09(0.01)$ & $0.22(0.03)$ & $0.09(0.01)$ & $-0.09(0.01)$ \\
\hline S.Korea-Czech R. & $0.80(0.33)$ & $-0.45(0.20)$ & $2.20(0.30)$ & $-1.64(0.35)$ & $-0.01(0.03)$ & $0.14(0.04)$ & $0.10(0.02)$ & $0.03(0.02)$ \\
\hline Mexico-Czech R. & $5.23(0.56)$ & $-7.47(0.85)$ & $-9.96(1.47)$ & $4.52(0.57)$ & $0.37(0.04)$ & $-0.53(0.05)$ & $-0.08(0.03)$ & $0.02(0.03)$ \\
\hline Norway-Czech R. & $2.31(0.59)$ & $-3.01(0.55)$ & $-1.82(0.93)$ & $2.80(0.63)$ & $-0.08(0.04)$ & $-0.24(0.08)$ & $0.11(0.06)$ & $0.03(0.05)$ \\
\hline Poland-Czech R. & $-1.32(0.28)$ & $1.27(0.41)$ & $1.15(0.44)$ & $0.66(0.32)$ & $-0.002(0.01)$ & $-0.08(0.02)$ & $0.03(0.03)$ & $0.16(0.03)$ \\
\hline Sweden-Czech R. & $4.95(0.61)$ & $-2.01(0.43)$ & $4.18(0.52)$ & $-3.74(0.49)$ & $-0.03(0.02)$ & $0.003(0.03)$ & $-0.05(0.03)$ & $0.12(0.02)$ \\
\hline Norway-Hungary & $2.57(0.97)$ & $-1.68(0.32)$ & $-0.60(0.35)$ & $1.50(0.83)$ & $0.07(0.05)$ & $-0.07(0.03)$ & $0.17(0.02)$ & $0.05(0.02)$ \\
\hline Norway-Iceland & $-0.13(0.54)$ & $0.24(0.21)$ & $4.69(0.88)$ & $-1.69(0.29)$ & $-0.07(0.03)$ & $0.14(0.02)$ & $-0.18(0.06)$ & $-0.21(0.03)$ \\
\hline Norway-Israel & $-1.29(0.75)$ & $0.58(0.52)$ & $2.25(0.39)$ & $2.34(0.42)$ & $-0.08(0.05)$ & $0.38(0.10)$ & $0.41(0.06)$ & $-0.43(0.13)$ \\
\hline Norway-S.Korea & $-0.14(0.26)$ & $0.22(0.35)$ & $-6.23(0.69)$ & $-1.75(0.52)$ & $-0.08(0.02)$ & $0.26(0.04)$ & $-0.11(0.05)$ & $0.25(0.04)$ \\
\hline Norway-Mexico & $-1.06(0.20)$ & $0.97(0.22)$ & $1.74(0.45)$ & $-0.70(0.63)$ & $0.08(0.01)$ & $-0.09(0.03)$ & $-0.02(0.04)$ & $0.11(0.04)$ \\
\hline Norway-Poland & $-0.96(0.23)$ & $1.08(0.16)$ & $1.84(0.29)$ & $-0.22(0.33)$ & $0.04(0.01)$ & $0.0002(0.02)$ & $0.03(0.02)$ & $0.02(0.02)$ \\
\hline Norway-Turkey & $1.73(1.39)$ & $1.07(0.55)$ & $0.28(1.63)$ & $-5.31(1.34)$ & $0.35(0.06)$ & $0.24(0.05)$ & $-0.12(0.09)$ & $-0.27(0.06)$ \\
\hline Sweden-Hungary & $-2.17(1.03)$ & $4.40(0.60)$ & $7.52(0.97)$ & $-10.6(1.01)$ & $-0.01(0.06)$ & $0.02(0.04)$ & $0.01(0.05)$ & $-0.12(0.03)$ \\
\hline Sweden-Iceland & $-13.9(6.91)$ & $8.73(1.71)$ & $-44.9(10.2)$ & $-5.37(2.19)$ & $-2.45(0.35)$ & $1.27(0.15)$ & $1.34(0.49)$ & $-1.74(0.25)$ \\
\hline Sweden-S.Korea & $2.93(0.64)$ & $-1.29(0.41)$ & $1.83(0.76)$ & $-4.57(0.60)$ & $0.13(0.09)$ & $-0.30(0.09)$ & $-0.22(0.06)$ & $-0.08(0.07)$ \\
\hline Sweden-Mexico & $0.05(0.92)$ & $-0.75(0.84)$ & $-4.83(1.04)$ & $2.84(2.57)$ & $0.06(0.03)$ & $0.35(0.05)$ & $0.11(0.07)$ & $-0.37(0.06)$ \\
\hline Sweden-Poland & $-12.4(4.89)$ & $4.42(2.23)$ & $11.6(2.32)$ & $10.8(3.36)$ & $-1.07(0.20)$ & $0.22(0.13)$ & $0.52(0.21)$ & $0.16(0.17)$ \\
\hline Sweden-Turkey & $0.52(0.46)$ & $-0.29(0.28)$ & $2.82(0.67)$ & $-1.06(0.65)$ & $-0.09(0.04)$ & $-0.14(0.02)$ & $0.001(0.03)$ & $0.14(0.02)$ \\
\hline
\end{tabular}

Note: Standard errors are given in parentheses. 
According to the results based on 40 pairs, only a few of them support the monetary model. However we should keep in mind that the most suitable monetary model version for a long-run analysis is FPMM, which fails to provide the theoretical relationships in every estimation. Thus, even the very weak support for the monetary model based on FLMM and RIDM is questionable, since derivation of RIDM reflects short term adjustment mechanism that makes the model inappropriate for a long-run analysis; while FLMM requires the conditions, rational expectations as well as absence of rational bubbles, which may be violated.

To reveal the reason for the failure of the monetary models, building blocks of the fundamental version (FPMM); PPP and KMDF are tested individually. According to (absolute) PPP expressed in (8), price level coefficients of the home and foreign countries have to be 1 and -1, respectively. However, it is sufficient to have the expected coefficient signs, irrespective of the magnitude, to accept that PPP holds due to the reasons effective on the magnitude such as; transportation cost, variety in the content of the price indices and differences in weights of the products forming the price indices.

PPP and KMDF are tested by employing the equations below that bring about FPMM;

$s=\gamma_{1} p+\gamma_{2} p^{*}$

$p=\gamma_{3} m+\gamma_{4} y+\gamma_{5} i^{\mathrm{L}}$

PPP is accepted if $\gamma_{1}$ and $\gamma_{2}$ are significantly positive and negative, respectively, regardless of their magnitudes. The validity of KMDF is supported if $\gamma_{3}$ and $\gamma_{5}$ are significantly positive, while $\gamma_{4}$ is significantly negative. Although KMDF employs real money balances (M/P), monetary model utilises money supply and price level individually. Thus, KMDF is tested in its decomposed version since we are interested in testing the building block of FPMM.

Long term interest rate is chosen as the relevant interest rate to be consistent with the monetary models, because FPMM is based on the idea that interest rate reflects expected inflation and that is assumed to be acceptable for the long term interest rate but not for the short term interest rate. Besides, it is not suitable to use short term interest rate, since RIDM incorporates it to the model as the remedy for short term deviations from PPP.

Table-4 provides Johansen cointegration results for PPP and decomposed version of KMDF. The numbers in columns headed $m, y, i^{L}, p, p^{*}$ correspond to the coefficient estimates of the variables. Standard errors are given in parentheses. The tables reveal that one of the main problems about monetary model is the invalidity of KMDF. PPP is supported for some of the country pairs, whereas KMDF holds only for Mexico. Thus, it is clear that the failure of the monetary model is not just due to deviations from PPP but it is also related with insufficient specification of money demand function. 


\section{TABLE-4}

Cointegration Test Results:

\begin{tabular}{|c|c|c|}
\hline \multicolumn{3}{|c|}{ Purchasing Power Parity } \\
\hline Home-Foreign & $p$ & $p^{*}$ \\
\hline Australia-USA & $-9.29(2.31)$ & $9.25(2.62)$ \\
\hline Canada-USA & $-1.76(3.65)$ & $-0.98(3.71)$ \\
\hline Chile-USA & \multicolumn{2}{|c|}{ No Cointegration } \\
\hline Czech R.-USA & \multicolumn{2}{|c|}{ No Cointegration } \\
\hline Denmark-USA & \multicolumn{2}{|c|}{ No Cointegration } \\
\hline Euro Area-USA & \multicolumn{2}{|c|}{ No Cointegration } \\
\hline Hungary-USA & $0.69(0.70)$ & $1.02(1.53)$ \\
\hline Iceland-USA $* * *$ & $5.28(0.83)$ & $-10.4(1.31)$ \\
\hline Israel-USA & \multicolumn{2}{|c|}{ No Cointegration } \\
\hline Japan-USA & $-42.1(5.59)$ & 2.89 (2.39) \\
\hline$\underline{\text { S. Korea-USA }}^{* * *}$ & $7.44(1.27)$ & $-9.58(1.55)$ \\
\hline$\underline{\text { Mexico-USA }}^{* * *}$ & $4.17(0.55)$ & $-5.70(1.12)$ \\
\hline New Zealand-USA & \multicolumn{2}{|c|}{ No Cointegration } \\
\hline Norway-USA & $-0.01(1.21)$ & $-0.89(0.94)$ \\
\hline Poland-USA & \multicolumn{2}{|c|}{ No Cointegration } \\
\hline Sweden-USA & \multicolumn{2}{|c|}{ No Cointegration } \\
\hline Switzerland-USA & \multicolumn{2}{|c|}{ No Cointegration } \\
\hline Turkey-USA & \multicolumn{2}{|c|}{ No Cointegration } \\
\hline UK-USA & \multicolumn{2}{|c|}{ No Cointegration } \\
\hline Japan-Euro Area & $-50.1(14.9)$ & $-6.48(1.68)$ \\
\hline$\underline{\text { UK-Euro Area }}^{* * *}$ & $2.29(1.07)$ & $-2.77(1.42)$ \\
\hline Hungary-Czech R. & $0.24(0.45)$ & $1.31(0.94)$ \\
\hline S.Korea-Czech R. & \multicolumn{2}{|c|}{ No Cointegration } \\
\hline Mexico-Czech R. & \multicolumn{2}{|c|}{ No Cointegration } \\
\hline Norway-Czech R. & $-443(96.6)$ & $337(70.4)$ \\
\hline Poland-Czech $R^{*}{ }^{* * *}$ & $2.34(0.48)$ & $-1.71(0.55)$ \\
\hline Sweden-Czech R. & \multicolumn{2}{|c|}{ No Cointegration } \\
\hline Norway-Hungary * & $1.02(1.03)$ & $-1.86(0.43)$ \\
\hline$\underline{\text { Norway-Iceland }}^{* * *}$ & $18.2(3.88)$ & $-6.34(1.38)$ \\
\hline Norway-Israel $* * *$ & $8.08(0.82)$ & $-5.13(0.86)$ \\
\hline Norway-S.Korea & \multicolumn{2}{|c|}{ No Cointegration } \\
\hline Norway-Mexico * & $0.54(2.24)$ & $-1.03(1.00)$ \\
\hline Norway-Poland & \multicolumn{2}{|c|}{ No Cointegration } \\
\hline Norway-Turkey & \multicolumn{2}{|c|}{ No Cointegration } \\
\hline Sweden-Hungary & $-1.46(0.72)$ & $-1.20(0.21)$ \\
\hline Sweden-Iceland & \multicolumn{2}{|c|}{ No Cointegration } \\
\hline$\underline{\text { Sweden-S.Korea }}^{* * *}$ & $9.35(3.00)$ & $-5.47(1.50)$ \\
\hline$\underline{\text { Sweden-Mexico }} * * *$ & $17.2(4.04)$ & $-7.79(1.31)$ \\
\hline$\underline{\text { Sweden-Poland }} * * *$ & $8.91(2.10)$ & $-5.00(1.04)$ \\
\hline Sweden-Turkey & \multicolumn{2}{|c|}{ No Cointegration } \\
\hline
\end{tabular}

Note: Standard errors are given in parentheses.

\begin{tabular}{|c|c|c|c|}
\hline \multicolumn{4}{|c|}{ (Decomposed) Keynesian Money Demand Function } \\
\hline Country & $m$ & $y$ & $i^{L}$ \\
\hline Australia & $0.24(0.11)$ & $1.01(0.35)$ & $0.06(0.01)$ \\
\hline Canada & $0.34(0.02)$ & $0.74(0.09)$ & $0.05(0.01)$ \\
\hline Chile & $0.10(0.04)$ & $1.57(0.26)$ & $0.06(0.02)$ \\
\hline Czech R. & $0.29(0.01)$ & $-0.20(0.04)$ & $-0.004(0.003)$ \\
\hline Denmark & \multicolumn{3}{|c|}{ No Cointegration } \\
\hline Euro Area & $0.21(0.01)$ & $-0.26(0.05)$ & $-0.03(0.01)$ \\
\hline Hungary & $1.94(0.79)$ & $-2.97(1.17)$ & $-0.46(0.08)$ \\
\hline Iceland & $0.06(0.01)$ & $0.37(0.04)$ & $-0.01(0.003)$ \\
\hline Israel & \multicolumn{3}{|c|}{ No Cointegration } \\
\hline Japan & $-0.22(0.03)$ & $-0.02(0.02)$ & $-0.04(0.01)$ \\
\hline S. Korea & $0.01(0.08)$ & $0.51(0.06)$ & $0.01(0.01)$ \\
\hline Mexico ${ }^{* * *}$ & $0.53(0.03)$ & $-0.57(0.13)$ & $0.02(0.004)$ \\
\hline New Zealand & $0.35(0.11)$ & $-2.17(0.53)$ & $-0.13(0.02)$ \\
\hline Norway & $0.06(0.02)$ & $-0.64(0.07)$ & $-0.001(0.004)$ \\
\hline Poland & $0.38(0.31)$ & $-1.02(0.76)$ & $-0.22(0.04)$ \\
\hline Sweden & $0.15(0.01)$ & $-0.04(0.04)$ & $-0.01(0.004)$ \\
\hline Switzerland & $0.48(0.14)$ & $0.29(0.26)$ & $0.21(0.03)$ \\
\hline Turkey & $0.33(0.04)$ & $0.40(0.13)$ & $-0.01(0.004)$ \\
\hline UK & $-1.46(0.32)$ & $1.95(1.47)$ & $-0.48(0.10)$ \\
\hline USA & $0.29(0.08)$ & $1.05(0.18)$ & $0.05(0.01)$ \\
\hline
\end{tabular}




\section{Concluding Remarks}

Modelling exchange rates has been an active area of research during the past three decades. In this research, popular variants of the monetary model of exchange rate determination; flexible price monetary model (FPMM), forward-looking monetary model (FLMM) and real interest differential model (RIDM) are tested for OECD member countries by utilising Johansen cointegration technique. Test results show that the models generally fail to establish the expected link between macroeconomic variables and exchange rates.

Researchers presume that the failure of the monetary model is mainly due to deviations from PPP. Thus, they come up with the extended versions that allow temporary or permanent deviations from PPP. However, test results reveal that (decomposed) Keynesian money demand function, one of the building blocks of the monetary model, is generally rejected as well. Thus, we suggest that to form an exchange rate determination model based on macro fundamentals, first, a valid money demand function must be formulated.

However, we expect that the monetary model will still be insufficient to determine the exchange rates of reserve currencies since expressing all supply and demand influences for reserve currencies with a few macroeconomic variables is not realistic. Besides, adding several components to the model may not be a good remedy since the power of econometric tests will decrease. We believe that there is a link between macroeconomic variables and exchange rates but the framework of the monetary model is not suitable to reveal it.

\section{Acknowledgements}

The authors thank two anonymous referees and the editor for their helpful comments and suggestions on a previous draft of this paper, substantially improving its content and quality. This paper is based upon work supported by the Government of Spain and FEDER under grant numbers ECO2011-23189 and ECO2013-48326. Simón Sosvilla-Rivero thanks the Universitat de Barcelona and RFA-IREA for their hospitality. Responsibility for any remaining errors rests with the authors. 


\section{References}

Baillie, R.T. and Selover, D.D. (1987) Cointegration and Models of Exchange Rate Determination, International Journal of Forecasting, 3(1), 43-51.

Bilson, J.F.O. (1978) Rational expectations and the exchange rate, The Economics of Exchange Rates in (Eds.) J. A. Frenkel and H. G. Johnson (eds), Addison-Wesley, Reading, MA, 75-96.

Cagan, P. (1956) The monetary dynamics of hyperinflation, Studies in the quantity theory of money, ed. by Friedman, M., 25-117, University of Chicago Press, Chicago.

Cerra, V. and Saxena, S. C. (2010) The monetary model strikes back: Evidence from the world, Journal of International Economics, 81, 184-196.

Cheung, Y.-W. and Chinn, M. D. (1998) Integration, Cointegration and the Forecast Consistency of Structural Exchange Rate Models, Journal of International Money and Finance, 17, 813-830.

Cheung, Y.-W., Chinn, M. D. and Garcia Pascual, A. (2005) Empirical exchange rate models of the nineties: are Any fit to survive?, Journal of International Money and Finance, 24, 1150-1175.

Chinn, M. D. and Meese, R. A. (1995) Banking on currency forecasts: How predictable is change in money, Journal of International Economics, 38, 161-178.

Dornbusch, R. (1976) Expectations and exchange rate dynamics, Journal of Political Economy, 84, 11611176.

Eichengreen, B. (2005) Sterling's past, Dollar's future: historical perspectives on reserve currency competition, National Bureau of Economic Research, Washigton, DC. Working Paper 11336.

Frankel, J.A. (1979) On the Mark: A theory of floating exchange rates based on real interest differentials, American Economic Review, 69, 610-622.

Frenkel, J. (1976) A monetary approach to the exchange rate: Doctrinal aspects and empirical evidence, Scandinavian Journal of Economics, 76, 200-224.

Groen, J.J.J. (2000) The monetary exchange rate model as a long-run phenomenon, Journal of International Economics, 52, 299-319.

Gujarati, D. N. and Porter, D. C. (2009) Basic Econometrics, McGraw Hill International Edition, 5, 741.

Helleiner, E. (2008) Political determinants of international currencies: what future for the US Dollar?, Review of International Political Economy, 15, 354-378.

Hooper, P. and Morton, J. (1982) Fluctuations in the Dollar: A Model of Nominal and Real Exchange Rate Determination, Journal of International Money and Finance, 1, 39-56.

Hunter, J. and Menla Ali, F. (2014) Money demand instability and real exchange rate persistence in the monetary model of USD-JPY exchange rate, Economic Modelling, 40, 42-51.

Husted, S. and MacDonald, R. (1998) Monetary-based models of the exchange rate: A panel perspective, Journal of International Financial Markets, Institutions and Money, 8, 1-19.

Huynh, K. P. and Schmidt-Dengler,P. (2014) The Role of Card Acceptance in the Transaction Demand for Money, Center for Economic Policy Research, London, Discussion Paper 10183.

Hwang, J.K. (2001) Dynamic forecasting of monetary exchange rate models: evidence from cointegration, International Advances in Economic Research, 7, 51-64. 
Johansen, S. (1988) Statistical analysis of cointegration vectors, Journal of Economic Dynamics and Control, 12, 231-254.

Johansen, S. and Juselius, K. (1990) Maximum likelihood estimation and inference on cointegration-with applications to the demand for money, Oxford Bulletin of Economics and Statistics, 52, 169-210.

Kearney, C. and MacDonald, R. (1990) Rational Expectations, Bubbles and Monetary Models of the Exchange Rate: The Australian/US dollar Rate during the Recent Float, Australian Economic Papers, 44, 1-20.

Ketenci, N. and Uz, I. (2008) Panel analysis of the monetary approach to exchange rates: Evidence from ten new EU members and Turkey, Emerging Markets Review, 9(1), 57-69.

Lucas, R. E. and Nicolini, J. P. (2015) On the Stability of Money Demand, Federal Reserve Bank of Minneapolis, Working Paper 718.

MacDonald, R. (2007) Exchange Rate Economics. Theories and evidence, Routledge, London.

MacDonald, R. and Taylor, M. P. (1992) The Monetary Approach to the Exchange Rate: Rational Expectations, Long-Run Equilibrium, and Forecasting, IMF Working Paper, 92/34.

MacDonald, R. and Taylor, M. P. (1994) The Monetary Model of the Exchange Rate: Long-run Relationships, Short-run Dynamics and How to Beat a Random Walk, Journal of International Money and Finance, 13, 276-90.

Meese, R. A. and Rogoff, K. (1983a) Empirical exchange rate models of the seventies: Do they fit out-ofsample?, Journal of International Economics, 14, 3-24.

Meese, R. A. and Rogoff, K. (1983b) The out-of-sample failure of empirical exchange rate models: sampling error or mis-specification?, Exchange Rates and International Macroeconomics, (Ed) J. Frenkel, University of Chicago Press, Chicago, 67-105

Mussa, M. (1976) The exchange rate, the balance of payments, and monetary and fiscal policy under a regime of controlled floating, Scandinavian Journal of Economics, 78, 229-248.

Neely, C. J. and Sarno, L. (2002) How Well Do Monetary Fundamentals Forecast Exchange Rates?, Federal Reserve Bank of St. Louis Review, 84(5), 51-74.

Phillips, P.C.B. (1987) Time Series Regression with a Unit Root, Econometrica, 55, 277-301.

Phillips, P.C.B. and Perron, P. (1988) Testing for a Unit Root in Time Series Regression, Biometrika, 75, 335-346.

Rapach, D.E. and Wohar, M.E. (2004) Testing the monetary model of exchange rate determination: a closer look at panels, Journal of International Money and Finance, 23, 867-895.

Sarno, L. and Taylor, M. P. (2002) The Economics of Exchange Rates, Cambridge University Press, Cambridge.

Smith, P and Wickens, M. (1984) An Empirical Investigation into the Causes of the Failure of the Monetary Model of the Exchange Rate, Centre for Economic Policy Research, London.

Sosvilla-Rivero, S. and García, E. (2006) Purchasing power parity revisited, International Macroeconomics: Recent Developments, (ed.) A. Morales Zumaquero, Nova Science Publishers, New York, 1-37. 


\section{Appendix - A}

\section{Data Description}

Monthly variables are used for all countries except Switzerland, Australia and New Zealand since some of the variables are available on a quarterly basis for those countries. Thus, quarterly averages of monthly variables are calculated to form the country pairs; Switzerland-USA, Australia-USA and New Zealand-USA. All the other country pairs are based on monthly data.

Industrial production and consumer price indices are obtained from OECD database for all countries. Base year is 2010 for both of the indices.

Exchange rates for the British Pound-Euro and the Japanese Yen-Euro are obtained from the European Central Bank, while central bank of Mexico provides the exchange rate for the Norwegian KroneMexican Peso. Central banks of Sweden, Norway and Czech Republic are the sources for the remaining exchange rates of Group-2 currency pairs. All the other exchange rates against the US Dollar (Group-1 currency pairs) are obtained from the web page of OECD.

M1 is used as the relevant money supply. IMF is the source for Canada and Denmark. Statistics Norway provides the data for Norway. All the other M1 data are obtained from each country's central bank.

Data for the short term and long term interest rates are mainly obtained from OECD except; Hungary: 3 month treasury bill and 10 year government bond rates are, respectively, the short and long term interest rates (Source: Central bank of Hungary).

Mexico: 3 year government bond rate is the long term interest rate (Source: Central bank of Mexico). Turkey: Average deposit rates up to 3 months and 1 year are, respectively, the short and long term interest rates (Source: Central bank of Turkey).

USA: 3 month treasury bill rate is the short term interest rate (Source: St Louis FED).

Research periods for all the models are presented below. $\mathrm{M}$ and $\mathrm{Q}$ refer, respectively, to month and quarter. In every country pair, first-named ones are the home countries and KMDF pertains to them. USA is the foreign country in the first 19 pairs below.

Australia-USA: All the models (Q1-1984/Q1-2014)

Canada-USA: PPP (M7-1970/M5-2014); all the other models (M7-1970/M4-2014)

Chile-USA: PPP, FLMM (M10-1999/M5-2014); FPMM, RIDM, KMDF (M7-2004 /M5-2014)

Czech Republic-USA: PPP (M1-2003/M5-2014); all the other models (M1-2003/M4-2014)

Denmark-USA: PPP (M1-1991/M5-2014); all the other models (M1-1991/M4-2014)

Euro Area-USA ${ }^{6}$ : PPP (M1-2002/M5-2014); all the other models (M1-2002/M4-2014)

Hungary-USA: PPP (M3-2008/M5-2014); all the other models (M3-2008/M4-2014)

\footnotetext{
${ }^{6}$ Physical euro coins and banknotes entered into circulation in January 2002. Thus, the analyses for Euro Area-USD and UK-Euro
} Area start from M1-2002, even though data is available for previous years. 
Iceland-USA: All the models (M4-2001/M8-2008)

Israel-USA: PPP (M7-2005/M5-2014); all the other models (M7-2005/M4-2014)

Japan-USA: PPP (M3-1973/M5-2014); all the other models (M4-2003/M4-2014)

S.Korea-USA: PPP(M1-1998/M6-2014); FPMM, RIDM, KMDF (M10-2000/M5-2014); FLMM(M1-1998/M5-2014)

Mexico-USA: PPP(M1-1995/M5-2014); FPMM,RIDM,KMDF(M10-2001/M4-2014);FLMM (M1-1995/M4-2014)

New Zealand-USA: All the models (Q2-1988/Q1-2014)

Norway-USA: PPP (M4-2001/M5-2014); all the other models (M4-2001/M4-2014)

Poland-USA: All the models (M1-2004/M5-2014)

Sweden-USA: PPP (M1-1998/M5-2014); all the other models (M1-1998/M4-2014)

Switzerland-USA: PPP (Q1-1985/Q1-2014); all the other models (Q1-1985/Q4-2013)

Turkey-USA: PPP (M1-2006/M6-2014); all the other models (M1-2006/M5-2014)

United Kingdom-USA: PPP (M10-1992/M5-2014); all the other models (M10-1992/M4-2014)

USA: KMDF (M7-1970/M5-2014)

Japan-Euro Area: PPP (M4-2003/M5-2014); all the other models (M4-2003/M4-2014)

United Kingdom-Euro Area: PPP (M1-2002/M5-2014); all the other models (M1-2002/M4-2014)

Hungary-Czech R.: PPP (M3-2008/M5-2014); all the other models (M3-2008/M4-2014)

S.Korea-Czech R.: PPP (M1-2004/M7-2014); all the other models (M1-2004/M4-2014)

Mexico-Czech R.: PPP (M1-2008/M5-2014); all the other models (M1-2008/M4-2014)

Norway-Czech R.: PPP (M1-2003/M5-2014); all the other models (M1-2003/M4-2014)

Poland-Czech R.: PPP (M1-2004/M5-2014); all the other models (M1-2004/M4-2014)

Sweden-Czech R.: PPP (M1-2003/M5-2014); all the other models (M1-2003/M4-2014)

Norway-Hungary: PPP (M3-2008/M5-2014); all the other models (M3-2008/M4-2014)

Norway-Iceland: All the models (M4-2001/M8-2008)

Norway-Israel: PPP (M1-2011/M5-2014); all the other models (M1-2011/M4-2014)

Norway-S.Korea: PPP (M4-2001/M5-2014); all the other models (M4-2001/M4-2014)

Norway-Mexico: PPP (M1-2002/M5-2014); all the other models (M1-2002/M4-2014)

Norway-Poland: PPP (M1-2004/M5-2014); all the other models (M1-2004/M4-2014)

Norway-Turkey: PPP (M1-2006/M5-2014); all the other models (M1-2006/M4-2014)

Sweden-Hungary: PPP (M3-2008/M5-2014); all the other models (M3-2008/M4-2014)

Sweden-Iceland: All the models (M4-2001/M8-2008)

Sweden-S.Korea: PPP (M9-2005/M5-2014); all the other models (M9-2005/M4-2014)

Sweden-Mexico: PPP (M1-1998/M5-2014); all the other models (M1-1998/M4-2014)

Sweden-Poland: PPP (M1-2004/M5-2014); all the other models (M1-2004/M4-2014)

Sweden-Turkey: PPP (M1-2006/M5-2014); all the other models (M9-2005/M4-2014) 


\section{Appendix - B}

\section{Unit Root Test Results}

The null hypothesis is: there is unit root. + and - refer, respectively, to acceptance and rejection of the null hypothesis at $5 \%$ significance level. c denotes constant, c\&T is constant and trend, $n$ indicates using none of them in the unit root tests. For each variable, only the results of the longest time series are presented.

\begin{tabular}{|c|c|c|c|c|c|c|c|c|c|c|c|c|c|c|c|c|c|c|}
\hline & \multicolumn{9}{|c|}{ LEVEL } & \multicolumn{9}{|c|}{ FIRST DIFFERENCE } \\
\hline & \multicolumn{6}{|c|}{ Augmented Dickey Fuller } & \multicolumn{3}{|c|}{ Philips Perron } & \multicolumn{6}{|c|}{ Augmented Dickey Fuller } & \multicolumn{3}{|c|}{ Philips Perron } \\
\hline & \multicolumn{3}{|c|}{ AIC } & \multicolumn{3}{|c|}{ SIC } & \multirow[b]{2}{*}{$\mathrm{n}$} & \multirow[b]{2}{*}{ C } & \multirow[b]{2}{*}{$\begin{array}{l}\mathrm{C} \\
\& \\
\mathrm{~T}\end{array}$} & \multicolumn{3}{|c|}{$\mathrm{AIC}$} & \multicolumn{3}{|c|}{$\mathrm{SIC}$} & \multirow[b]{2}{*}{$\mathrm{n}$} & \multirow[b]{2}{*}{ c } & \multirow[b]{2}{*}{$\begin{array}{l}\mathrm{C} \\
\& \\
\mathrm{~T}\end{array}$} \\
\hline & $\mathrm{n}$ & c & $\begin{array}{l}\mathrm{c} \\
\& \\
T\end{array}$ & $\mathrm{n}$ & $\mathrm{c}$ & $\begin{array}{l}\mathrm{C} \\
\& \\
T\end{array}$ & & & & $\mathrm{n}$ & $\mathrm{c}$ & $\begin{array}{l}\mathrm{C} \\
\& \\
\mathrm{~T}\end{array}$ & $n$ & $\mathrm{C}$ & $\begin{array}{l}\mathrm{C} \\
\& \\
T\end{array}$ & & & \\
\hline \multicolumn{19}{|l|}{ Australia } \\
\hline (USD) $\mathrm{s}$ & + & + & + & + & + & + & + & + & + & - & - & - & - & - & - & - & - & - \\
\hline $\mathrm{m}$ & + & - & + & + & + & + & + & + & + & + & - & - & - & - & - & - & - & - \\
\hline $\mathrm{y}$ & + & + & + & + & + & + & + & + & + & - & - & - & - & - & - & - & - & - \\
\hline$i^{S}$ & + & + & + & + & + & + & + & + & + & - & - & - & - & - & - & - & - & - \\
\hline$i^{L}$ & - & + & + & - & + & + & - & + & + & - & - & - & - & - & - & - & - & - \\
\hline$p$ & + & - & - & + & - & - & + & - & + & + & + & - & + & - & - & - & - & - \\
\hline & & & & & & & & & & & & & & & & & & \\
\hline \multicolumn{19}{|l|}{ Canada } \\
\hline (USD) $\mathrm{s}$ & + & + & + & + & + & + & + & + & + & - & - & - & - & - & - & - & - & - \\
\hline $\mathrm{m}$ & + & + & + & + & + & + & + & + & + & - & - & - & - & - & - & - & - & - \\
\hline$y$ & + & + & + & + & + & + & + & + & + & - & - & - & - & - & - & - & - & - \\
\hline$i^{s}$ & + & + & - & + & + & + & + & + & + & - & - & - & - & - & - & - & - & - \\
\hline$i^{L}$ & + & + & + & + & + & + & + & + & + & - & - & - & - & - & - & - & - & - \\
\hline$p$ & + & - & + & + & - & + & + & - & + & - & - & - & + & - & - & - & - & - \\
\hline \multicolumn{19}{|l|}{ Chile } \\
\hline (USD) $\mathrm{s}$ & + & + & + & + & + & + & + & + & + & - & - & - & - & - & - & - & - & - \\
\hline $\mathrm{m}$ & + & + & + & + & + & + & + & + & + & + & - & - & - & - & - & - & - & - \\
\hline$y$ & + & + & + & + & + & + & + & + & - & - & - & - & - & - & - & - & - & - \\
\hline$i^{5}$ & + & + & + & + & + & + & + & + & + & - & - & - & - & - & - & - & - & - \\
\hline$i^{L}$ & + & + & + & + & + & + & + & + & + & - & - & - & - & - & - & - & - & - \\
\hline$p$ & + & + & + & + & + & + & + & + & + & - & - & - & - & - & - & - & - & - \\
\hline \multicolumn{19}{|l|}{ Czech R. } \\
\hline (USD) $\mathrm{s}$ & + & + & + & + & + & + & + & + & + & - & - & - & - & - & - & - & - & - \\
\hline $\mathrm{m}$ & + & + & + & + & - & + & + & + & + & + & - & - & + & - & - & - & - & - \\
\hline$y$ & + & + & + & + & + & + & + & + & + & - & - & - & - & - & - & - & - & - \\
\hline$i^{s}$ & + & + & + & + & + & + & + & + & + & - & - & - & - & - & - & - & - & - \\
\hline$i^{L}$ & + & + & + & + & + & + & + & + & + & - & - & - & - & - & - & - & - & - \\
\hline$p$ & + & + & + & + & + & + & + & + & + & - & - & - & - & - & - & - & - & - \\
\hline Denmark & & & & & & & & & & & & & & & & & & \\
\hline (USD) $\mathrm{s}$ & + & + & + & + & + & + & + & + & + & - & - & - & - & - & - & - & - & - \\
\hline $\mathrm{m}$ & + & + & + & + & + & + & + & + & + & - & - & - & - & - & - & - & - & - \\
\hline$y$ & + & + & + & + & + & + & + & + & + & - & - & - & - & - & - & - & - & - \\
\hline$i^{S}$ & - & + & + & - & + & + & - & + & + & - & - & - & - & - & - & - & - & - \\
\hline$i^{L}$ & + & + & - & - & + & - & - & + & - & - & - & - & - & - & - & - & - & - \\
\hline$p$ & + & + & + & + & + & + & + & + & + & + & - & - & - & - & - & - & - & - \\
\hline
\end{tabular}




\begin{tabular}{|c|c|c|c|c|c|c|c|c|c|c|c|c|c|c|c|c|c|c|}
\hline & \multicolumn{9}{|c|}{ LEVEL } & \multicolumn{9}{|c|}{ FIRST DIFFERENCE } \\
\hline & \multicolumn{6}{|c|}{ Augmented Dickey Fuller } & \multicolumn{3}{|c|}{ Philips Perron } & \multicolumn{6}{|c|}{ Augmented Dickey Fuller } & \multicolumn{3}{|c|}{ Philips Perron } \\
\hline & \multicolumn{3}{|c|}{ AIC } & \multicolumn{3}{|c|}{ SIC } & \multirow[b]{2}{*}{$\mathrm{n}$} & \multirow[b]{2}{*}{ c } & \multirow[b]{2}{*}{$\begin{array}{l}\mathrm{C} \\
\& \\
\mathrm{~T}\end{array}$} & \multicolumn{3}{|c|}{ AIC } & \multicolumn{3}{|c|}{ SIC } & \multirow[b]{2}{*}{$\mathrm{n}$} & \multirow[b]{2}{*}{ C } & \multirow[b]{2}{*}{$\begin{array}{l}\mathrm{C} \\
\& \\
\mathrm{~T}\end{array}$} \\
\hline & $\mathrm{n}$ & $\mathrm{C}$ & $\begin{array}{l}\mathrm{C} \\
\& \\
\mathrm{~T}\end{array}$ & $\mathrm{n}$ & $\mathrm{C}$ & $\begin{array}{l}c \\
\& \\
T\end{array}$ & & & & $\mathrm{n}$ & c & $\begin{array}{l}\mathrm{C} \\
\& \\
T\end{array}$ & $\mathrm{n}$ & $\mathrm{C}$ & $\begin{array}{l}C \\
\& \\
T\end{array}$ & & & \\
\hline \multicolumn{19}{|l|}{ Euro Area } \\
\hline (USD) $\mathrm{s}$ & + & - & + & + & - & + & + & - & + & - & - & - & - & - & - & - & - & - \\
\hline $\mathrm{m}$ & + & + & + & + & + & + & + & + & + & - & - & - & - & - & - & - & - & - \\
\hline$y$ & + & - & + & + & - & + & + & + & + & - & - & - & - & - & - & - & - & - \\
\hline$i^{s}$ & + & + & + & + & + & + & + & + & + & - & - & - & - & - & - & - & - & - \\
\hline$i^{L}$ & + & + & + & + & + & + & + & + & + & - & - & - & - & - & - & - & - & - \\
\hline$p$ & + & + & - & + & + & + & + & + & + & + & - & - & - & - & - & - & - & - \\
\hline Hungary & & & & & & & & & & & & & & & & & & \\
\hline (USD) $\mathrm{s}$ & - & + & - & + & - & - & + & + & + & - & - & - & - & - & - & - & - & - \\
\hline $\mathrm{m}$ & + & + & + & + & + & + & + & + & + & - & - & - & - & - & - & - & - & - \\
\hline$y$ & + & + & + & + & + & + & + & + & + & - & - & - & - & - & - & - & - & - \\
\hline$i^{s}$ & + & + & + & + & + & + & + & + & + & - & - & + & - & - & - & - & - & - \\
\hline$i^{L}$ & + & + & + & + & + & + & + & + & + & - & - & - & - & - & - & - & - & - \\
\hline$p$ & + & + & + & + & - & + & + & + & + & + & + & - & + & - & - & - & - & - \\
\hline Iceland & & & & & & & & & & & & & & & & & & \\
\hline (USD) $\mathrm{s}$ & + & + & + & + & + & + & + & + & + & - & - & - & - & - & - & - & - & - \\
\hline $\mathrm{m}$ & + & + & + & + & + & + & + & + & + & - & - & - & - & - & - & - & - & - \\
\hline$y$ & + & + & + & + & + & + & + & + & + & - & - & - & - & - & - & - & - & - \\
\hline$i^{s}$ & + & + & + & + & + & + & + & + & + & - & - & - & - & - & - & - & - & - \\
\hline$i^{L}$ & + & + & + & + & + & + & + & + & + & - & - & - & - & - & - & - & - & - \\
\hline$p$ & + & + & + & + & + & + & + & + & + & - & - & - & - & - & - & - & - & - \\
\hline Israel & & & & & & & & & & & & & & & & & & \\
\hline (USD) $\mathrm{s}$ & + & + & + & + & + & + & + & + & + & - & - & - & - & - & - & - & - & - \\
\hline $\mathrm{m}$ & + & + & + & + & + & + & + & + & + & - & - & - & - & - & - & - & - & - \\
\hline$y$ & + & + & + & + & + & - & + & + & - & - & - & - & - & - & - & - & - & - \\
\hline$i^{s}$ & - & + & + & + & + & + & + & + & + & - & - & + & - & - & - & - & - & - \\
\hline$i^{L}$ & - & + & - & + & + & - & + & + & + & - & - & - & - & - & - & - & - & - \\
\hline$p$ & + & + & + & + & + & + & + & + & + & - & - & - & - & - & - & - & - & - \\
\hline Japan & & & & & & & & & & & & & & & & & & \\
\hline (USD) $\mathrm{s}$ & + & + & + & + & + & + & + & + & + & - & - & - & - & - & - & - & - & - \\
\hline $\mathrm{m}$ & + & + & + & + & + & + & + & + & + & - & - & - & - & - & - & - & - & - \\
\hline$y$ & + & + & + & + & + & + & + & + & + & - & - & - & - & - & - & - & - & - \\
\hline$i^{5}$ & + & + & + & + & + & + & + & + & + & - & - & - & - & - & - & - & - & - \\
\hline$i^{L}$ & + & + & - & + & + & - & + & + & - & - & - & - & - & - & - & - & - & - \\
\hline$p$ & + & - & - & + & - & - & + & - & - & - & - & - & - & - & - & - & - & - \\
\hline S. Korea & & & & & & & & & & & & & & & & & & \\
\hline (USD) $\mathrm{s}$ & + & + & + & + & - & + & + & - & + & - & - & - & - & - & - & - & - & - \\
\hline $\mathrm{m}$ & + & + & + & + & + & + & + & + & + & - & - & - & - & - & - & - & - & - \\
\hline$y$ & + & + & + & + & + & + & + & + & + & - & - & - & - & - & - & - & - & - \\
\hline$i^{s}$ & + & + & + & + & + & + & + & + & + & - & - & - & - & - & - & - & - & - \\
\hline$i^{L}$ & + & + & + & - & + & + & - & + & + & - & - & - & - & - & - & - & - & - \\
\hline$p$ & + & + & + & + & + & - & + & + & + & + & - & - & - & - & - & - & - & - \\
\hline
\end{tabular}




\begin{tabular}{|c|c|c|c|c|c|c|c|c|c|c|c|c|c|c|c|c|c|c|}
\hline & \multicolumn{9}{|c|}{ LEVEL } & \multicolumn{9}{|c|}{ FIRST DIFFERENCE } \\
\hline & \multicolumn{6}{|c|}{ Augmented Dickey Fuller } & \multicolumn{3}{|c|}{ Philips Perron } & \multicolumn{6}{|c|}{ Augmented Dickey Fuller } & \multicolumn{3}{|c|}{ Philips Perron } \\
\hline & \multicolumn{3}{|c|}{ AIC } & \multicolumn{3}{|c|}{ SIC } & \multirow[b]{2}{*}{$\mathrm{n}$} & \multirow[b]{2}{*}{ c } & \multirow[b]{2}{*}{$\begin{array}{l}\mathrm{C} \\
\& \\
\mathrm{~T}\end{array}$} & \multicolumn{3}{|c|}{ AIC } & \multicolumn{3}{|c|}{ SIC } & \multirow[b]{2}{*}{$\mathrm{n}$} & \multirow[b]{2}{*}{ c } & \multirow[b]{2}{*}{$\begin{array}{l}\mathrm{C} \\
\& \\
\mathrm{~T}\end{array}$} \\
\hline & $\mathrm{n}$ & c & $\begin{array}{l}\mathrm{C} \\
\& \\
T\end{array}$ & $\mathrm{n}$ & c & $\begin{array}{l}c \\
\& \\
T\end{array}$ & & & & $\mathrm{n}$ & c & $\begin{array}{l}c \\
\& \\
T\end{array}$ & $\mathrm{n}$ & c & $\begin{array}{l}\mathrm{C} \\
\& \\
T\end{array}$ & & & \\
\hline \multicolumn{19}{|l|}{ Mexico } \\
\hline (USD) $\mathrm{s}$ & + & + & - & + & - & - & + & - & - & - & - & - & - & - & - & - & - & - \\
\hline $\mathrm{m}$ & + & - & - & + & - & - & + & - & + & - & - & - & - & - & - & - & - & - \\
\hline y & + & + & - & + & - & - & + & - & - & - & - & - & - & - & - & - & - & - \\
\hline$i^{s}$ & + & + & + & + & + & + & + & + & + & - & - & - & - & - & - & - & - & - \\
\hline$i^{L}$ & - & + & - & - & + & - & - & + & - & - & - & - & - & - & - & - & - & - \\
\hline $\mathrm{p}$ & + & + & - & + & - & - & + & - & - & + & - & - & - & - & - & - & - & - \\
\hline New Zealand & & & & & & & & & & & & & & & & & & \\
\hline (USD) $\mathrm{s}$ & + & + & + & + & + & + & + & + & + & - & - & - & - & - & - & - & - & - \\
\hline $\mathrm{m}$ & + & + & + & + & + & + & + & + & + & - & - & - & - & - & - & - & - & - \\
\hline y & + & $\begin{array}{lll}+ & & \end{array}$ & + & + & + & + & + & + & + & - & - & - & - & - & - & - & - & - \\
\hline$i^{5}$ & - & + & + & - & + & + & - & + & + & - & - & - & - & - & - & - & - & - \\
\hline$i^{L}$ & - & $\begin{array}{llll}+ & & & \end{array}$ & + & - & + & + & - & + & + & - & - & - & - & - & - & - & - & - \\
\hline $\mathrm{p}$ & + & + & + & + & + & + & + & + & + & - & - & - & - & - & - & - & - & - \\
\hline Norway & & & & & & & & & & - & - & & - & & & & & \\
\hline (USD) $\mathrm{s}$ & + & + & + & + & + & + & + & + & + & - & - & - & - & - & - & - & - & - \\
\hline $\mathrm{m}$ & + & + & + & + & + & + & + & + & + & - & - & + & - & - & + & - & - & - \\
\hline$y$ & + & + & - & + & + & - & + & + & - & - & - & - & - & - & - & - & - & - \\
\hline & + & + & + & + & + & + & + & + & + & - & - & - & - & - & - & - & - & - \\
\hline & + & + & + & + & + & + & + & + & + & - & - & - & - & - & - & - & - & - \\
\hline & + & + & - & + & + & - & + & + & + & - & - & - & - & - & - & - & - & - \\
\hline Poland & & & & & & & & & & & & & & & & & & \\
\hline (USD) $\mathrm{s}$ & + & - & - & + & - & + & + & + & + & - & - & - & - & - & - & - & - & - \\
\hline $\mathrm{m}$ & + & + & + & + & + & + & + & + & + & + & - & - & - & - & - & - & - & - \\
\hline$y$ & + & + & + & + & + & + & + & + & + & - & - & - & - & - & - & - & - & - \\
\hline$i^{5}$ & + & + & + & + & + & + & + & + & + & - & - & - & - & - & - & - & - & - \\
\hline$i^{L}$ & + & + & + & + & + & + & + & + & + & - & - & - & - & - & - & - & - & - \\
\hline & + & + & + & + & + & + & + & + & + & - & - & - & - & - & - & - & - & - \\
\hline Sweden & & & & & & & & & & & & & & & & & & \\
\hline (USD) $\mathrm{s}$ & + & + & + & + & + & + & + & + & + & - & - & - & - & - & - & - & - & - \\
\hline $\mathrm{m}$ & + & + & + & + & + & + & + & + & + & - & - & - & - & - & - & - & - & - \\
\hline$y$ & + & + & + & + & + & + & + & + & + & - & - & - & - & - & - & - & - & - \\
\hline & + & + & + & + & + & + & + & + & + & - & - & - & - & - & - & - & - & - \\
\hline$i^{L}$ & + & + & - & + & + & + & + & + & + & - & - & - & - & - & - & - & - & - \\
\hline & + & + & + & + & + & + & + & + & + & - & - & - & - & - & - & - & - & - \\
\hline Switzerland & & & & & & & & & & & & & & & & & & \\
\hline (USD) $\mathrm{s}$ & + & + & + & - & + & + & - & + & + & - & - & - & - & - & - & - & - & - \\
\hline $\mathrm{m}$ & + & + & + & + & + & + & + & + & + & - & - & - & - & - & - & - & - & - \\
\hline$y$ & + & + & + & + & + & + & + & + & + & - & - & - & - & - & - & - & - & - \\
\hline$i^{5}$ & + & + & + & + & + & + & + & + & + & - & - & - & - & - & - & - & - & - \\
\hline & + & + & + & + & + & + & + & + & + & - & - & - & - & - & - & - & - & - \\
\hline$p$ & + & - & + & + & + & + & + & - & + & + & + & + & - & - & - & - & - & - \\
\hline
\end{tabular}




\begin{tabular}{|c|c|c|c|c|c|c|c|c|c|c|c|c|c|c|c|c|c|c|}
\hline & \multicolumn{9}{|c|}{ LEVEL } & \multicolumn{9}{|c|}{ FIRST DIFFERENCE } \\
\hline & \multicolumn{6}{|c|}{ Augmented Dickey Fuller } & \multicolumn{3}{|c|}{ Philips Perron } & \multicolumn{6}{|c|}{ Augmented Dickey Fuller } & \multicolumn{3}{|c|}{ Philips Perron } \\
\hline & \multicolumn{3}{|c|}{$\mathrm{AIC}$} & \multicolumn{3}{|c|}{ SIC } & \multirow[b]{2}{*}{$\mathrm{n}$} & \multirow[b]{2}{*}{$\mathrm{c}$} & \multirow[b]{2}{*}{$\begin{array}{l}\mathrm{C} \\
\& \\
\mathrm{~T}\end{array}$} & \multicolumn{3}{|c|}{$\mathrm{AIC}$} & \multicolumn{3}{|c|}{ SIC } & \multirow[b]{2}{*}{$\mathrm{n}$} & \multirow[b]{2}{*}{ C } & \multirow[b]{2}{*}{$\begin{array}{l}\mathrm{C} \\
\& \\
\mathrm{~T}\end{array}$} \\
\hline & $\mathrm{n}$ & c & $\begin{array}{l}\mathrm{C} \\
\& \\
\mathrm{~T}\end{array}$ & $\mathrm{n}$ & $\mathrm{c}$ & $\begin{array}{l}\mathrm{C} \\
\& \\
\mathrm{~T}\end{array}$ & & & & $n$ & c & $\begin{array}{l}\mathrm{C} \\
\& \\
T \\
\end{array}$ & $n$ & $\mathrm{c}$ & $\begin{array}{l}\mathrm{C} \\
\& \\
T\end{array}$ & & & \\
\hline Turkey & & & & & & & & & & & & & & & & & & \\
\hline (USD) $\mathrm{s}$ & + & + & + & + & + & + & + & + & + & - & - & - & - & - & - & - & - & - \\
\hline $\mathrm{m}$ & + & + & + & + & + & + & + & + & + & + & - & - & - & - & - & - & - & - \\
\hline $\mathrm{y}$ & + & + & + & + & + & + & + & + & + & - & - & - & - & - & - & - & - & - \\
\hline$i^{S}$ & + & + & + & + & + & + & + & + & + & - & - & - & - & - & - & - & - & - \\
\hline$i^{L}$ & + & + & + & + & + & + & + & + & + & - & - & - & - & - & - & - & - & - \\
\hline$p$ & + & + & + & + & + & + & + & + & + & + & - & - & - & - & - & - & - & - \\
\hline UK & & & & & & & & & & & & & & & & & & \\
\hline (USD) $\mathrm{s}$ & + & + & + & + & + & + & + & + & + & - & - & - & - & - & - & - & - & - \\
\hline $\mathrm{m}$ & + & + & + & + & + & + & + & + & + & + & - & - & - & - & - & - & - & - \\
\hline $\mathrm{y}$ & + & + & + & + & + & + & + & + & + & - & - & - & - & - & - & - & - & - \\
\hline$i^{s}$ & + & + & + & + & + & + & + & + & + & - & - & - & - & - & - & - & - & - \\
\hline$i^{L}$ & + & + & + & + & + & + & + & + & + & - & - & - & - & - & - & - & - & - \\
\hline$p$ & + & + & + & + & + & + & + & + & + & + & - & - & - & - & - & - & - & - \\
\hline USA & & & & & & & & & & & & & & & & & & \\
\hline $\mathrm{m}$ & + & + & + & + & + & + & + & + & + & - & - & - & - & - & - & - & - & - \\
\hline$y$ & + & + & + & + & + & + & + & + & + & - & - & - & - & - & - & - & - & - \\
\hline$i^{5}$ & + & + & + & + & + & + & + & + & + & - & - & - & - & - & - & - & - & - \\
\hline$i^{L}$ & + & + & + & + & + & + & + & + & + & - & - & - & - & - & - & - & - & - \\
\hline$p$ & + & - & + & + & - & + & + & - & + & - & - & - & - & - & - & - & - & - \\
\hline Japan-Euro Area & + & + & + & + & + & + & + & + & + & - & - & - & - & - & - & - & - & - \\
\hline UK-Euro Area & + & + & + & + & + & + & + & + & + & - & - & - & - & - & - & - & - & - \\
\hline Hungary-Czech R. s & + & + & + & + & + & + & + & + & + & - & - & - & - & - & - & - & - & - \\
\hline S.Korea-Czech R. $\quad$ s & + & + & + & + & + & + & + & + & + & - & - & - & - & - & - & - & - & - \\
\hline Mexico-Czech R. $\mathrm{s}$ & + & - & - & + & - & - & + & - & - & - & - & - & - & - & - & - & - & - \\
\hline Norway-Czech R. $\mathrm{s}$ & + & + & + & + & + & + & + & + & + & - & - & - & - & - & - & - & - & - \\
\hline $\begin{array}{ll}\text { Poland-Czech R. } \quad \mathrm{s} \\
\end{array}$ & + & + & + & + & + & + & + & + & + & - & - & - & - & - & - & - & - & - \\
\hline Sweden-Czech R. $\quad$ s & + & + & + & + & + & + & + & + & + & - & - & - & - & - & - & - & - & - \\
\hline Norway-Hungary s & + & + & + & + & + & + & + & + & + & - & - & - & - & - & - & - & - & - \\
\hline Norway-Iceland & + & + & + & + & + & + & + & + & + & - & - & - & - & - & - & - & - & - \\
\hline Norway-Israel & + & + & + & + & + & + & + & + & + & - & - & - & - & - & - & - & - & - \\
\hline Norway-S.Korea & + & + & + & + & + & + & + & + & + & - & - & - & - & - & - & - & - & - \\
\hline Norway-Mexico & + & - & - & + & - & - & + & - & - & - & - & - & - & - & - & - & - & - \\
\hline Norway-Poland & + & + & + & + & + & + & + & + & + & - & - & - & - & - & - & - & - & - \\
\hline Norway-Turkey & + & + & - & + & + & - & - & + & + & - & - & - & - & - & - & - & - & - \\
\hline Sweden-Hungary $\mathrm{s}$ & + & + & + & + & + & + & + & + & + & - & - & - & - & - & - & - & - & - \\
\hline Sweden-Iceland $\mathrm{s}$ & + & + & + & + & + & + & + & + & + & - & - & - & - & - & - & - & - & - \\
\hline Sweden-S.Korea $\quad \mathrm{s}$ & + & + & + & + & + & + & + & + & + & - & - & - & - & - & - & - & - & - \\
\hline Sweden-Mexico $\quad \mathrm{s}$ & + & + & + & + & + & + & + & + & + & - & - & - & - & - & - & - & - & - \\
\hline Sweden-Poland & + & + & + & + & + & + & + & + & + & - & - & - & - & - & - & - & - & - \\
\hline Sweden-Turkey & + & + & + & + & + & + & - & + & + & - & - & - & - & - & - & - & - & - \\
\hline
\end{tabular}




\section{Appendix - C}

Trace Test Results: Flexible Price Monetary Model

\begin{tabular}{|c|c|c|c|c|c|c|c|}
\hline Home-Foreign & $r=0$ & $r \leq 1$ & $r \leq 2$ & $r \leq 3$ & $r \leq 4$ & $r \leq 5$ & $r \leq 6$ \\
\hline Australia-USA & $143.4(0.00)$ & $94.2(0.06)$ & $58.7(0.27)$ & $32.9(0.56)$ & $16.2(0.69)$ & $6.0(0.69)$ & $0.0(0.86)$ \\
\hline Canada-USA & $240.7(0.00)$ & $139.1(0.00)$ & $80.9(0.00)$ & $37.4(0.32)$ & $18.1(0.55)$ & $5.7(0.72)$ & $0.2(0.66)$ \\
\hline Chile-USA & $167.3(0.00)$ & $121.4(0.00)$ & $83.2(0.00)$ & $52.2(0.01)$ & $26.7(0.10)$ & $10.0(0.28)$ & $1.3(0.24)$ \\
\hline Czech R.-USA & $168.3(0.00)$ & $95.8(0.04)$ & $62.1(0.17)$ & $37.6(0.32)$ & $15.5(0.74)$ & $4.0(0.90)$ & $0.2(0.67)$ \\
\hline Denmark-USA & $164.8(0.00)$ & $111.6(0.00)$ & $67.2(0.07)$ & $36.2(0.38)$ & $13.6(0.85)$ & $5.3(0.77)$ & $0.0(0.81)$ \\
\hline Euro Area-USA & $177.4(0.00)$ & $111.0(0.00)$ & $72.5(0.02)$ & $40.6(0.19)$ & $21.2(0.34)$ & $7.9(0.47)$ & $1.7(0.19)$ \\
\hline Hungary-USA & $150.0(0.00)$ & $101.9(0.01)$ & $67.5(0.07)$ & $40.2(0.21)$ & $17.7(0.58)$ & $6.6(0.62)$ & $0.6(0.44)$ \\
\hline Iceland-USA & $129.0(0.03)$ & $94.5(0.06)$ & $65.5(0.10)$ & $46.6(0.06)$ & $28.9(0.06)$ & $13.2(0.10)$ & $3.6(0.05)$ \\
\hline Israel-USA & $165.2(0.00)$ & $104.4(0.01)$ & $67.2(0.07)$ & $41.9(0.16)$ & $17.8(0.58)$ & $7.5(0.51)$ & $1.2(0.27)$ \\
\hline Japan-USA & $178.7(0.00)$ & $126.8(0.00)$ & $82.0(0.00)$ & $49.9(0.03)$ & $20.5(0.38)$ & $7.9(0.47)$ & $0.5(0.45)$ \\
\hline S.Korea-USA & $192.3(0.00)$ & $119.6(0.00)$ & $76.3(0.01)$ & $52.7(0.01)$ & $31.6(0.03)$ & $15.8(0.04)$ & $2.4(0.11)$ \\
\hline Mexico-USA & $145.8(0.00)$ & $100.3(0.02)$ & $64.5(0.12)$ & $37.3(0.33)$ & $15.2(0.76)$ & $6.8(0.59)$ & $0.1(0.75)$ \\
\hline New Zealand-USA & $156.1(0.00)$ & $87.3(0.16)$ & $57.3(0.32)$ & $33.4(0.52)$ & $15.7(0.72)$ & $8.8(0.38)$ & $3.1(0.07)$ \\
\hline Norway-USA & $195.6(0.00)$ & $132.0(0.00)$ & $86.8(0.00)$ & $44.1(0.10)$ & $22.8(0.25)$ & $8.7(0.38)$ & $0.2(0.63)$ \\
\hline Poland-USA & $166.8(0.00)$ & $106.2(0.00)$ & $73.9(0.02)$ & $46.5(0.06)$ & $24.0(0.19)$ & $10.0(0.27)$ & $0.0(0.92)$ \\
\hline Sweden-USA & $142.3(0.00)$ & $89.9(0.11)$ & $51.4(0.57)$ & $29.9(0.72)$ & $12.6(0.90)$ & $4.3(0.87)$ & $0.2(0.64)$ \\
\hline Switzerland-USA & $145.2(0.00)$ & $92.6(0.08)$ & $51.9(0.55)$ & $30.2(0.70)$ & $15.6(0.74)$ & $4.7(0.83)$ & $0.4(0.53)$ \\
\hline Turkey-USA & $220.7(0.00)$ & $153.1(0.00)$ & $92.4(0.00)$ & $46.5(0.06)$ & $24.3(0.18)$ & $9.8(0.29)$ & $0.0(0.99)$ \\
\hline UK-USA & $187.6(0.00)$ & $121.9(0.00)$ & $63.7(0.13)$ & $36.3(0.37)$ & $16.2(0.69)$ & $7.7(0.49)$ & $2.9(0.08)$ \\
\hline Japan-Euro Area & $165.1(0.00)$ & $113.6(0.00)$ & $68.1(0.06)$ & $36.4(0.37)$ & $14.8(0.78)$ & $2.8(0.97)$ & $0.0(0.95)$ \\
\hline UK-Euro Area & $179.3(0.00)$ & $111.9(0.00)$ & $73.6(0.02)$ & $38.1(0.29)$ & $16.2(0.69)$ & $7.8(0.48)$ & $1.5(0.20)$ \\
\hline Hungary-Czech R. & $170.3(0.00)$ & $87.6(0.15)$ & $56.4(0.35)$ & $31.0(0.66)$ & $16.6(0.66)$ & $3.9(0.90)$ & $0.03(0.85)$ \\
\hline S.Korea-Czech R. & $158.3(0.00)$ & $110.0(0.00)$ & $75.3(0.01)$ & $43.1(0.12)$ & $20.2(0.40)$ & $7.6(0.50)$ & $0.6(0.41)$ \\
\hline Mexico- Czech R. & $128.8(0.03)$ & $80.7(0.33)$ & $51.2(0.58)$ & $33.1(0.54)$ & $19.5(0.45)$ & $8.1(0.45)$ & $0.0(0.87)$ \\
\hline Norway-Czech R. & $149.0(0.00)$ & $93.5(0.07)$ & $59.4(0.25)$ & $30.8(0.67)$ & $17.0(0.63)$ & $5.8(0.71)$ & $0.1(0.70)$ \\
\hline Poland-Czech R. & $146.4(0.00)$ & $84.7(0.22)$ & $55.0(0.41)$ & $35.8(0.40)$ & $20.2(0.40)$ & $9.1(0.35)$ & $0.4(0.49)$ \\
\hline Sweden-Czech R. & $179.1(0.00)$ & $97.5(0.03)$ & $51.8(0.55)$ & $29.8(0.72)$ & $13.7(0.85)$ & $5.3(0.77)$ & $1.5(0.21)$ \\
\hline Norway-Hungary & $148.5(0.00)$ & $105.5(0.00)$ & $64.3(0.12)$ & $34.3(0.48)$ & $18.3(0.53)$ & $5.4(0.75)$ & $0.0(0.86)$ \\
\hline Norway-Iceland & $205.9(0.00)$ & $141.1(0.00)$ & $93.3(0.00)$ & $49.5(0.03)$ & $31.0(0.03)$ & $14.5(0.06)$ & $2.5(0.10)$ \\
\hline Norway-Israel & $152.7(0.00)$ & $103.3(0.01)$ & $63.3(0.14)$ & $38.8(0.26)$ & $20.0(0.42)$ & $9.6(0.31)$ & $0.6(0.40)$ \\
\hline Norway-S.Korea & $149.1(0.00)$ & $84.5(0.23)$ & $51.4(0.57)$ & $34.9(0.44)$ & $22.2(0.28)$ & $10.9(0.21)$ & $4.6(0.03)$ \\
\hline Norway-Mexico & $166.5(0.00)$ & $107.0(0.00)$ & $70.5(0.04)$ & $40.6(0.20)$ & $21.9(0.29)$ & $7.3(0.53)$ & $1.5(0.21)$ \\
\hline Norway-Poland & $155.7(0.00)$ & $91.2(0.09)$ & $57.0(0.33)$ & $32.6(0.57)$ & $13.9(0.84)$ & $5.8(0.71)$ & $1.6(0.20)$ \\
\hline Norway-Turkey & $144.9(0.00)$ & $99.1(0.02)$ & $67.5(0.07)$ & $40.3(0.20)$ & $18.5(0.52)$ & $3.0(0.96)$ & $0.0(0.90)$ \\
\hline Sweden-Hungary & $176.7(0.00)$ & $106.5(0.00)$ & $64.7(0.11)$ & $38.0(0.29)$ & $17.2(0.61)$ & $7.0(0.57)$ & $1.1(0.28)$ \\
\hline Sweden-Iceland & $113.6(0.21)$ & $76.9(0.47)$ & $51.4(0.57)$ & $30.1(0.71)$ & $15.9(0.71)$ & $7.1(0.56)$ & $0.5(0.46)$ \\
\hline Sweden-S.Korea & $148.8(0.00)$ & $88.2(0.14)$ & $54.8(0.42)$ & $31.3(0.64)$ & $18.0(0.56)$ & $6.1(0.67)$ & $0.9(0.33)$ \\
\hline Sweden-Mexico & $144.5(0.00)$ & $91.9(0.08)$ & $56.4(0.35)$ & $26.1(0.88)$ & $8.1(0.99)$ & $3.1(0.96)$ & $0.0(0.92)$ \\
\hline Sweden-Poland & $148.0(0.00)$ & $107.5(0.00)$ & $73.9(0.02)$ & $41.4(0.17)$ & $18.2(0.55)$ & $7.9(0.46)$ & $1.3(0.25)$ \\
\hline Sweden-Turkey & $143.3(0.00)$ & $102.6(0.01)$ & $66.8(0.08)$ & $33.5(0.52)$ & $19.3(0.46)$ & $8.6(0.39)$ & $0.4(0.51)$ \\
\hline
\end{tabular}

Note: $r$ denotes the number of cointegrating vectors. $p$-values are given in parantheses. 
Trace Test Results: Forward-Looking Monetary Model

\begin{tabular}{|c|c|c|c|c|c|}
\hline Home-Foreign & $\mathrm{r}=0$ & $\mathrm{r} \leq 1$ & $r \leq 2$ & $\mathrm{r} \leq 3$ & $\mathrm{r} \leq 4$ \\
\hline Australia-USA & $61.7(0.18)$ & $28.4(0.79)$ & $14.9(0.78)$ & $6.8(0.59)$ & $0.0(0.98)$ \\
\hline Canada-USA & $102.5(0.00)$ & $62.6(0.00)$ & $28.8(0.06)$ & $7.6(0.50)$ & $1.5(0.21)$ \\
\hline Chile-USA & $86.4(0.00)$ & $44.6(0.09)$ & $22.9(0.24)$ & $7.6(0.50)$ & $0.3(0.55)$ \\
\hline Czech R.-USA & $110.6(0.00)$ & $47.5(0.05)$ & $18.8(0.50)$ & $5.9(0.69)$ & $0.0(0.85)$ \\
\hline Denmark-USA & $87.7(0.00)$ & $44.2(0.10)$ & $14.0(0.83)$ & $5.5(0.75)$ & $0.0(0.90)$ \\
\hline Euro Area-USA & $96.5(0.00)$ & $58.2(0.00)$ & $33.7(0.01)$ & $14.4(0.07)$ & $0.7(0.39)$ \\
\hline Hungary-USA & $81.7(0.00)$ & $45.1(0.08)$ & $26.6(0.11)$ & $10.6(0.23)$ & $0.7(0.38)$ \\
\hline Iceland-USA & $86.0(0.00)$ & $54.8(0.00)$ & $27.1(0.09)$ & $10.6(0.23)$ & $2.7(0.09)$ \\
\hline Israel-USA & $84.6(0.00)$ & $49.1(0.03)$ & $26.0(0.12)$ & $11.1(0.20)$ & $0.4(0.49)$ \\
\hline Japan-USA & $96.9(0.00)$ & $54.9(0.00)$ & $21.8(0.30)$ & $10.4(0.24)$ & $0.8(0.36)$ \\
\hline S.Korea-USA & $119.0(0.00)$ & $76.7(0.00)$ & $35.9(0.00)$ & $10.4(0.24)$ & $0.6(0.41)$ \\
\hline Mexico-USA & $135.3(0.00)$ & $61.1(0.00)$ & $30.0(0.04)$ & $12.8(0.12)$ & $0.4(0.52)$ \\
\hline New Zealand-USA & $88.9(0.00)$ & $43.2(0.12)$ & $21.3(0.33)$ & $9.4(0.32)$ & $2.1(0.14)$ \\
\hline Norway-USA & $106.2(0.00)$ & $57.7(0.00)$ & $25.8(0.13)$ & $3.6(0.92)$ & $0.0(0.89)$ \\
\hline Poland-USA & $74.1(0.02)$ & $43.5(0.11)$ & $19.3(0.46)$ & $4.7(0.83)$ & $0.1(0.72)$ \\
\hline Sweden-USA & $76.8(0.01)$ & $33.5(0.52)$ & $14.0(0.83)$ & $5.0(0.80)$ & $0.1(0.67)$ \\
\hline Switzerland-USA & $84.3(0.00)$ & $46.6(0.06)$ & $17.5(0.60)$ & $7.2(0.54)$ & $0.3(0.59)$ \\
\hline Turkey-USA & $118.7(0.00)$ & $63.1(0.00)$ & $32.9(0.02)$ & $9.5(0.31)$ & $0.6(0.44)$ \\
\hline UK-USA & $73.2(0.02)$ & $37.2(0.33)$ & $16.2(0.69)$ & $7.0(0.57)$ & $2.1(0.14)$ \\
\hline Japan-Euro Area & $94.3(0.00)$ & $50.3(0.02)$ & $23.7(0.21)$ & $6.1(0.67)$ & $0.0(0.85)$ \\
\hline UK-Euro Area & $94.6(0.00)$ & $45.4(0.08)$ & $24.5(0.17)$ & $9.2(0.34)$ & $2.3(0.12)$ \\
\hline Hungary-Czech R. & $100.1(0.00)$ & $38.5(0.27)$ & $15.2(0.76)$ & $4.1(0.89)$ & $0.2(0.67)$ \\
\hline S.Korea-Czech R. & $90.9(0.00)$ & $54.0(0.01)$ & $24.1(0.19)$ & $9.1(0.35)$ & $0.3(0.57)$ \\
\hline Mexico-Czech R. & $75.5(0.01)$ & $43.0(0.13)$ & $16.9(0.64)$ & $5.8(0.70)$ & $0.0(0.91)$ \\
\hline Norway-Czech R. & $111.8(0.00)$ & $63.4(0.00)$ & $24.7(0.16)$ & $4.6(0.84)$ & $2.1(0.14)$ \\
\hline Poland-Czech R. & $76.5(0.01)$ & $30.9(0.67)$ & $12.6(0.90)$ & $6.0(0.69)$ & $0.7(0.40)$ \\
\hline Sweden-Czech R. & $127.5(0.00)$ & $56.6(0.00)$ & $20.4(0.39)$ & $5.5(0.75)$ & $1.8(0.17)$ \\
\hline Norway-Hungary & $78.8(0.00)$ & $38.7(0.27)$ & $17.7(0.58)$ & $5.0(0.80)$ & $0.3(0.55)$ \\
\hline Norway-Iceland & $89.0(0.00)$ & $45.0(0.08)$ & $24.6(0.17)$ & $8.4(0.41)$ & $0.1(0.68)$ \\
\hline Norway-Israel & $77.0(0.01)$ & $37.6(0.31)$ & $18.4(0.53)$ & $9.3(0.33)$ & $3.0(0.28)$ \\
\hline Norway-S.Korea & $97.9(0.00)$ & $45.0(0.08)$ & $23.3(0.23)$ & $11.6(0.17)$ & $3.3(0.06)$ \\
\hline Norway-Mexico & $85.7(0.00)$ & $52.1(0.01)$ & $23.2(0.23)$ & $5.3(0.77)$ & $1.2(0.26)$ \\
\hline Norway-Poland & $98.3(0.00)$ & $44.2(0.10)$ & $20.5(0.38)$ & $7.9(0.46)$ & $2.7(0.09)$ \\
\hline Norway-Turkey & $83.3(0.00)$ & $45.6(0.07)$ & $24.4(0.18)$ & $5.0(0.79)$ & $0.0(0.90)$ \\
\hline Sweden-Hungary & $95.5(0.00)$ & $41.8(0.16)$ & $18.8(0.50)$ & $9.3(0.33)$ & $2.0(0.15)$ \\
\hline Sweden-Iceland & $74.4(0.02)$ & $43.2(0.12)$ & $20.5(0.38)$ & $6.1(0.68)$ & $0.4(0.48)$ \\
\hline Sweden-S.Korea & $95.8(0.00)$ & $50.8(0.02)$ & $23.1(0.24)$ & $9.5(0.31)$ & $0.5(0.45)$ \\
\hline Sweden-Mexico & $47.3(0.74)$ & $23.6(0.94)$ & $11.4(0.95)$ & $4.7(0.83)$ & $0.2(0.62)$ \\
\hline Sweden-Poland & $81.1(0.00)$ & $51.3(0.02)$ & $23.8(0.20)$ & $9.6(0.31)$ & $3.7(0.05)$ \\
\hline Sweden-Turkey & $74.3(0.02)$ & $41.8(0.16)$ & $19.6(0.45)$ & $7.1(0.56)$ & $0.0(0.87)$ \\
\hline
\end{tabular}

Note: $r$ denotes the number of cointegrating vectors. $p$-values are given in parantheses. 
Trace Test Results: Real Interest Differential Model

\begin{tabular}{|c|c|c|c|c|c|c|c|c|c|}
\hline Home-Foreign & $r=0$ & $r \leq 1$ & $r \leq 2$ & $r \leq 3$ & $\mathrm{r} \leq 4$ & $\mathrm{r} \leq 5$ & $r \leq 6$ & $\mathrm{r} \leq 7$ & $r \leq 8$ \\
\hline Australia-USA & $272(0.00)$ & $184(0.00)$ & $136(0.00)$ & $96.8(0.04)$ & $58.2(0.29)$ & $33.1(0.55)$ & $15.6(0.73)$ & $6.4(0.63)$ & $0.3(0.56$ \\
\hline Canada-USA & $368(0.00)$ & $257(0.00)$ & $181(0.00)$ & $115(0.00)$ & $70.0(0.04)$ & $38.0(0.30)$ & $16.8(0.65)$ & $6.1(0.67)$ & $0.3(0.56$ \\
\hline Chile-USA & $292(0.00)$ & $174(0.00)$ & $129(0.03)$ & $87.4(0.16)$ & $55.4(0.39)$ & $31.8(0.62)$ & $11.5(0.94)$ & $4.1(0.88)$ & $0.1(0.69$ \\
\hline Czech R.-USA & $278(0.00)$ & $189(0.00)$ & $128(0.03)$ & $83.6(0.25)$ & $53.4(0.48)$ & $29.8(0.72)$ & $16.3(0.68)$ & $4.9(0.81)$ & $0.4(0.53$ \\
\hline Denmark-USA & $309(0.00)$ & $223(0.00)$ & $149(0.00)$ & $92.5(0.08)$ & $45.6(0.80)$ & $26.4(0.87)$ & $10.7(0.96)$ & $3.1(0.95)$ & $0.6(0.44$ \\
\hline Euro Area-USA & $291(0.00)$ & $207(0.00)$ & $136(0.00)$ & $92.2(0.08)$ & $52.8(0.51)$ & $32.4(0.58)$ & $17.8(0.57)$ & $4.5(0.85)$ & $0.0(0.81)$ \\
\hline Hungary-USA & $443(0.00)$ & $315(0.00)$ & $218(0.00)$ & $154(0.00)$ & $100(0.00)$ & $53.9(0.01)$ & $25.9(0.13)$ & $9.8(0.29)$ & $1.5(0.21)$ \\
\hline Iceland-USA & $252(0.00)$ & $180(0.00)$ & $130(0.02)$ & $91.7(0.09)$ & $55.8(0.38)$ & $34.4(0.47)$ & $20.9(0.36)$ & $8.1(0.45)$ & $2.5(0.11)$ \\
\hline Israel-USA & $249(0.00)$ & $184(0.00)$ & $136(0.00)$ & $92.2(0.08)$ & $65.1(0.11)$ & $40.4(0.20)$ & $22.6(0.26)$ & $7.3(0.53)$ & $0.1(0.74$ \\
\hline Japan-USA & $389(0.00)$ & $277(0.00)$ & $187(0.00)$ & $120(0.00)$ & $76.1(0.01)$ & $43.3(0.12)$ & $23.7(0.21)$ & $10.0(0.27)$ & $1.8(0.17$ \\
\hline S.Korea-USA & $291(0.00)$ & $207(0.00)$ & $145(0.00)$ & $99.3(0.02)$ & $60.0(0.23)$ & $37.4(0.32)$ & $22.9(0.24)$ & $9.6(0.30)$ & $3.4(0.06$ \\
\hline Mexico-USA & $266(0.00)$ & $201(0.00)$ & $143(0.00)$ & $98.5(0.03)$ & $61.1(0.20)$ & $30.9(0.66)$ & $12.4(0.91)$ & $3.3(0.94)$ & $0.0(0.88$ \\
\hline New Zealand-USA & $248(0.00)$ & $169(0.01)$ & $126(0.04)$ & $89.2(0.12)$ & $58.6(0.27)$ & $33.3(0.53)$ & $16.8(0.65)$ & $9.0(0.36)$ & $3.7(0.05$ \\
\hline Norway-USA & $297(0.00)$ & $225(0.00)$ & $170(0.00)$ & $116(0.00)$ & $72.3(0.03)$ & $38.2(0.29)$ & $22.2(0.28)$ & $9.2(0.34)$ & $0.1(0.74$ \\
\hline Poland-USA & $294(0.00)$ & $218(0.00)$ & $166(0.00)$ & $116(0.00)$ & $77.9(0.00)$ & $47.3(0.05)$ & $21.9(0.30)$ & $5.2(0.78)$ & $0.2(0.61)$ \\
\hline Sweden-USA & $255(0.00)$ & $190(0.00)$ & $132(0.01)$ & $85.9(0.19)$ & $48.7(0.69)$ & $29.3(0.75)$ & $17.9(0.56)$ & $8.6(0.39)$ & $0.0(0.84$ \\
\hline Switzerland-USA & $256(0.00)$ & $186(0.00)$ & $138(0.00)$ & $97.2(0.03)$ & $61.6(0.18)$ & $36.6(0.36)$ & $16.6(0.66)$ & $5.5(0.74)$ & $0.0(0.87$ \\
\hline Turkey-USA & $350(0.00)$ & $272(0.00)$ & $199(0.00)$ & $135(0.00)$ & $83.5(0.00)$ & $46.4(0.06)$ & $26.7(0.10)$ & $12.5(0.13)$ & $0.2(0.60$ \\
\hline UK-USA & $321(0.00)$ & $219(0.00)$ & $144(0.00)$ & $92.9(0.07)$ & $49.0(0.67)$ & $22.9(0.96)$ & $9.6(0.98)$ & $4.3(0.87)$ & $1.2(0.27$ \\
\hline Japan-Euro Area & $399(0.00)$ & $252(0.00)$ & $163(0.00)$ & $111(0.00)$ & $73.1(0.02)$ & $45.3(0.08)$ & $24.3(0.18)$ & $9.5(0.31)$ & $0.3(0.55$ \\
\hline UK-Euro Area & $263(0.00)$ & $180(0.00)$ & $132(0.01)$ & $97.1(0.04)$ & $66.2(0.09)$ & $45.2(0.08)$ & $26.4(0.11)$ & $15.0(0.05)$ & $5.8(0.01)$ \\
\hline Hungary-Czech R. & $827(0.00)$ & $633(0.00)$ & $485(0.00)$ & $357(0.00)$ & $252(0.00)$ & $153(0.00)$ & $87.7(0.00)$ & $29.3(0.00)$ & $3.2(0.07$ \\
\hline S.Korea-Czech R. & $288(0.00)$ & $226(0.00)$ & $167(0.00)$ & $114(0.00)$ & $74.5(0.02)$ & $43.9(0.11)$ & $26.4(0.11)$ & $10.8(0.21)$ & $0.6(0.41$ \\
\hline Mexico-Czech R. & $297(0.00)$ & $208(0.00)$ & $137(0.00)$ & $91.3(0.09)$ & $56.8(0.34)$ & $28.4(0.79)$ & $16.5(0.67)$ & $4.9(0.81)$ & $0.2(0.61)$ \\
\hline Norway-Czech R. & $281(0.00)$ & $211(0.00)$ & $142(0.00)$ & $92.4(0.08)$ & $50.4(0.61)$ & $26.7(0.86)$ & $14.7(0.79)$ & $6.6(0.62)$ & $0.5(0.44$ \\
\hline Poland-Czech R. & $293(0.00)$ & $214(0.00)$ & $148(0.00)$ & $101(0.01)$ & $68.3(0.06)$ & $38.8(0.26)$ & $20.1(0.41)$ & $9.8(0.28)$ & $2.0(0.15$ \\
\hline Sweden-Czech R. & $249(0.00)$ & $172(0.00)$ & $122(0.07)$ & $85.6(0.20)$ & $51.8(0.55)$ & $32.0(0.60)$ & $15.2(0.76)$ & $5.8(0.71)$ & $1.6(0.20$ \\
\hline Norway-Hungary & $328(0.00)$ & $230(0.00)$ & $159(0.00)$ & $117(0.00)$ & $79.9(0.00)$ & $51.7(0.02)$ & $25.5(0.14)$ & $10.0(0.28)$ & $0.8(0.35$ \\
\hline Norway-Iceland & $254(0.00)$ & $180(0.00)$ & $128(0.03)$ & $82.6(0.28)$ & $54.9(0.42)$ & $32.0(0.61)$ & $18.2(0.54)$ & $8.8(0.38)$ & $0.9(0.32$ \\
\hline Norway-Israel & $249(0.00)$ & $184(0.00)$ & $127(0.03)$ & $88.8(0.13)$ & $57.5(0.32)$ & $34.5(0.47)$ & $20.8(0.36)$ & $8.9(0.37)$ & $0.1(0.76$ \\
\hline Norway-S.Korea & $273(0.00)$ & $195(0.00)$ & $139(0.00)$ & $87.5(0.16)$ & $60.5(0.22)$ & $36.6(0.36)$ & $21.5(0.32)$ & $10.2(0.26)$ & $2.4(0.11)$ \\
\hline Norway-Mexico & $302(0.00)$ & $225(0.00)$ & $167(0.00)$ & $116(0.00)$ & $76.2(0.01)$ & $49.6(0.03)$ & $27.0(0.10)$ & $11.8(0.16)$ & $1.2(0.26$ \\
\hline Norway-Poland & $295(0.00)$ & $219(0.00)$ & $151(0.00)$ & $98.5(0.03)$ & $63.7(0.13)$ & $34.7(0.45)$ & $18.8(0.50)$ & $8.4(0.42)$ & $3.2(0.07$ \\
\hline Norway-Turkey & $260(0.00)$ & $196(0.00)$ & $140(0.00)$ & $94.1(0.06)$ & $64.9(0.11)$ & $40.8(0.19)$ & $22.4(0.27)$ & $5.9(0.70)$ & $0.7(0.40$ \\
\hline Sweden-Hungary & $294(0.00)$ & $213(0.00)$ & $153(0.00)$ & $100(0.02)$ & $69.3(0.05)$ & $40.9(0.18)$ & $21.1(0.35)$ & $7.9(0.47)$ & $1.0(0.31$ \\
\hline Sweden-Iceland & $229(0.00)$ & $158(0.05)$ & $113(0.22)$ & $77.8(0.43)$ & $51.4(0.57)$ & $32.2(0.59)$ & $20.7(0.37)$ & $9.9(0.28)$ & $0.8(0.36$ \\
\hline Sweden-S.Korea & $268(0.00)$ & $196(0.00)$ & $134(0.01)$ & $83.3(0.26)$ & $52.2(0.53)$ & $28.4(0.79)$ & $15.7(0.72)$ & $6.1(0.67)$ & $0.7(0.39$ \\
\hline Sweden-Mexico & $251(0.00)$ & $190(0.00)$ & $136(0.00)$ & $96.0(0.04)$ & $63.0(0.15)$ & $33.7(0.51)$ & $17.7(0.58)$ & $3.6(0.93)$ & $0.0(0.94$ \\
\hline Sweden-Poland & $269(0.00)$ & $193(0.00)$ & $133(0.01)$ & $96.4(0.04)$ & $64.7(0.11)$ & $39.3(0.24)$ & $20.4(0.39)$ & $8.2(0.44)$ & $0.6(0.42$ \\
\hline Sweden-Turkey & $245(0.00)$ & $176(0.00)$ & $128(0.03)$ & $90.8(0.10)$ & $55.5(0.39)$ & $34.3(0.48)$ & $14.4(0.81)$ & $4.1(0.89)$ & $0.0(0.78$ \\
\hline
\end{tabular}

Note: $r$ denotes the number of cointegrating vectors. $p$-values are given in parantheses. 
Trace Test Results: Purchasing Power Parity

\begin{tabular}{|c|c|c|c|}
\hline Home-Foreign & $\mathrm{r}=0$ & $\mathrm{r} \leq 1$ & $r \leq 2$ \\
\hline Australia-USA & $36.8(0.00)$ & $14.4(0.07)$ & $2.6(0.10)$ \\
\hline Canada-USA & $53.4(0.00)$ & $3.8(0.91)$ & $0.3(0.57)$ \\
\hline Chile-USA & $24.0(0.19)$ & $8.9(0.36)$ & $1.6(0.20)$ \\
\hline Czech R.-USA & $19.7(0.44)$ & $4.8(0.82)$ & $0.7(0.39)$ \\
\hline Denmark-USA & $20.3(0.40)$ & $6.8(0.60)$ & $1.5(0.21)$ \\
\hline Euro Area-USA & $26.3(0.11)$ & $10.2(0.26)$ & $2.5(0.10)$ \\
\hline Hungary-USA & $33.1(0.02)$ & $9.2(0.34)$ & $2.9(0.08)$ \\
\hline Iceland-USA & $36.2(0.00)$ & $11.4(0.18)$ & $0.6(0.42)$ \\
\hline Israel-USA & $15.5(0.74)$ & $3.3(0.94)$ & $0.5(0.45)$ \\
\hline Japan-USA & $156(0.00)$ & $28.0(0.00)$ & $8.3(0.00)$ \\
\hline S. Korea-USA & $43.6(0.00)$ & $20.5(0.00)$ & $0.4(0.49)$ \\
\hline Mexico-USA & $74.8(0.00)$ & $22.6(0.00)$ & $0.9(0.31)$ \\
\hline New Zealand-USA & $24.8(0.16)$ & $8.8(0.38)$ & $0.4(0.53)$ \\
\hline Norway-USA & $30.2(0.04)$ & $7.3(0.53)$ & $0.6(0.43)$ \\
\hline Poland-USA & $27.8(0.08)$ & $6.1(0.67)$ & $2.4(0.12)$ \\
\hline Sweden-USA & $23.0(0.24)$ & $7.6(0.50)$ & $0.3(0.54)$ \\
\hline Switzerland-USA & $23.6(0.21)$ & $10.5(0.24)$ & $0.1(0.73)$ \\
\hline Turkey-USA & $21.4(0.32)$ & $8.6(0.39)$ & $0.2(0.61)$ \\
\hline UK-USA & $18.6(0.52)$ & $6.0(0.68)$ & $0.0(0.94)$ \\
\hline Japan-Euro Area & $35.2(0.01)$ & $13.9(0.08)$ & $3.4(0.06)$ \\
\hline UK-Euro Area & $42.0(0.00)$ & $6.3(0.65)$ & $0.3(0.56)$ \\
\hline Hungary-Czech R. & $37.4(0.00)$ & $9.9(0.28)$ & $2.4(0.11)$ \\
\hline S.Korea-Czech R. & $28.2(0.07)$ & $9.3(0.33)$ & $2.6(0.10)$ \\
\hline Mexico- Czech R. & $27.2(0.09)$ & $10.2(0.26)$ & $1.6(0.19)$ \\
\hline Norway-Czech R. & $35.2(0.01)$ & $13.4(0.10)$ & $1.7(0.19)$ \\
\hline Poland-Czech R. & $37.9(0.00)$ & $12.6(0.12)$ & $2.0(0.15)$ \\
\hline Sweden-Czech R. & $18.4(0.53)$ & $5.3(0.76)$ & $0.9(0.32)$ \\
\hline Norway-Hungary & $31.7(0.02)$ & $8.8(0.38)$ & $1.9(0.15)$ \\
\hline Norway- Iceland & $33.5(0.01)$ & $16.6(0.03)$ & $5.7(0.01)$ \\
\hline Norway-Israel & $31.3(0.03)$ & $4.2(0.87)$ & $0.2(0.60)$ \\
\hline Norway- S.Korea & $14.0(0.83)$ & $6.8(0.59)$ & $1.6(0.20)$ \\
\hline Norway-Mexico & $41.7(0.00)$ & $18.6(0.01)$ & $1.0(0.30)$ \\
\hline Norway-Poland & $21.0(0.35)$ & $7.1(0.55)$ & $1.7(0.19)$ \\
\hline Norway-Turkey & $27.6(0.08)$ & $7.4(0.52)$ & $0.5(0.45)$ \\
\hline Sweden-Hungary & $34.0(0.01)$ & $8.6(0.39)$ & $3.0(0.08)$ \\
\hline Sweden-Iceland & $19.7(0.43)$ & $9.4(0.32)$ & $0.8(0.35)$ \\
\hline Sweden- S.Korea & $36.2(0.00)$ & $9.4(0.32)$ & $2.5(0.11)$ \\
\hline Sweden-Mexico & $57.2(0.00)$ & $14.8(0.06)$ & $5.1(0.02)$ \\
\hline Sweden-Poland & $31.8(0.02)$ & $13.2(0.10)$ & $2.5(0.11)$ \\
\hline Sweden-Turkey & $15.7(0.73)$ & $4.6(0.84)$ & $0.0(0.90)$ \\
\hline
\end{tabular}

Trace Test Results: Keynesian Money Demand Function

\begin{tabular}{rcccc}
\hline Country & $\mathrm{r}=0$ & $\mathrm{r} \leq 1$ & $\mathrm{r} \leq 2$ & $\mathrm{r} \leq 3$ \\
\hline Australia & $103(0.00)$ & $31.6(0.03)$ & $11.7(0.17)$ & $3.9(0.04)$ \\
Canada & $148(0.00)$ & $42.2(0.00)$ & $16.7(0.03)$ & $0.1(0.68)$ \\
Chile & $51.8(0.02)$ & $19.9(0.42)$ & $5.7(0.72)$ & $0.0(0.89)$ \\
Czech R. & $62.2(0.00)$ & $32.7(0.02)$ & $12.3(0.14)$ & $0.8(0.35)$ \\
Denmark & $38.3(0.28)$ & $14.9(0.78)$ & $7.0(0.56)$ & $2.7(0.09)$ \\
Euro Area & $80.8(0.00)$ & $30.9(0.03)$ & $15.0(0.05)$ & $3.5(0.06)$ \\
Hungary & $52.7(0.01)$ & $22.3(0.28)$ & $5.5(0.74)$ & $1.7(0.18)$ \\
Iceland & $68.4(0.00)$ & $36.7(0.00)$ & $14.4(0.07)$ & $3.7(0.05)$ \\
Israel & $38.4(0.28)$ & $19.6(0.44)$ & $10.5(0.24)$ & $1.5(0.21)$ \\
Japan & $86.6(0.00)$ & $35.3(0.01)$ & $14.5(0.06)$ & $0.0(0.84)$ \\
S. Korea & $51.9(0.01)$ & $31.0(0.03)$ & $12.4(0.13)$ & $1.9(0.16)$ \\
Mexico & $55.3(0.00)$ & $24.5(0.17)$ & $6.2(0.67)$ & $1.6(0.19)$ \\
New Zealand & $57.3(0.00)$ & $19.5(0.45)$ & $8.1(0.45)$ & $1.3(0.25)$ \\
Norway & $64.8(0.00)$ & $27.9(0.08)$ & $5.5(0.75)$ & $1.7(0.18)$ \\
Poland & $51.8(0.02)$ & $23.5(0.21)$ & $9.8(0.29)$ & $2.9(0.08)$ \\
Sweden & $59.3(0.00)$ & $25.1(0.15)$ & $7.5(0.51)$ & $0.1(0.68)$ \\
Switzerland & $62.0(0.00)$ & $19.4(0.45)$ & $7.0(0.57)$ & $0.2(0.61)$ \\
Turkey & $57.3(0.00)$ & $22.7(0.26)$ & $9.0(0.36)$ & $0.2(0.64)$ \\
UK & $54.6(0.01)$ & $26.6(0.11)$ & $10.6(0.23)$ & $0.0(0.91)$ \\
USA & $82.1(0.00)$ & $35.6(0.00)$ & $4.4(0.86)$ & $0.6(0.43)$ \\
\hline & & & &
\end{tabular}

Note: $r$ denotes the number of cointegrating vectors. $p$-values are given in parantheses. 